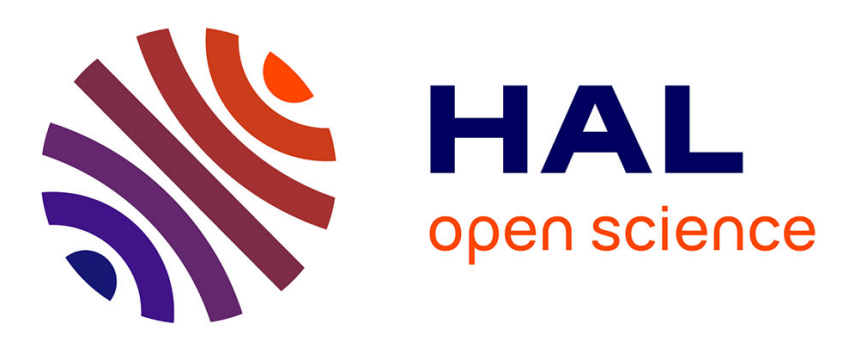

\title{
Évolution des propriétés magnétiques d'une substance polycristalline soumise à des cycles d'hystérésis dissymétriques successifs. Aspect directionnel du phénomène de reptation
}

\author{
J.L. Porteseil, R. Vergne
}

\section{To cite this version:}

J.L. Porteseil, R. Vergne. Évolution des propriétés magnétiques d'une substance polycristalline soumise à des cycles d'hystérésis dissymétriques successifs. Aspect directionnel du phénomène de reptation. Revue de Physique Appliquée, 1977, 12 (8), pp.1077-1093. 10.1051/rphysap:019770012080107700 . jpa-00244284

\author{
HAL Id: jpa-00244284 \\ https://hal.science/jpa-00244284
}

Submitted on 1 Jan 1977

HAL is a multi-disciplinary open access archive for the deposit and dissemination of scientific research documents, whether they are published or not. The documents may come from teaching and research institutions in France or abroad, or from public or private research centers.
L'archive ouverte pluridisciplinaire HAL, est destinée au dépôt et à la diffusion de documents scientifiques de niveau recherche, publiés ou non, émanant des établissements d'enseignement et de recherche français ou étrangers, des laboratoires publics ou privés. 


\title{
REVUE DE PHYSIQUE APPLIQUÉE
}

\author{
Classification \\ Physics Abstracts
} 8.550

\section{ÉVOLUTION DES PROPRIÉTÉS MAGNÉTIQUES D'UNE SUBSTANCE POLYCRISTALLINE SOUMISE A DES CYCLES D'HYSTÉRÉSIS DISSYMÉTRIQUES SUCCESSIFS ASPECT DIRECTIONNEL DU PHÉNOMÈNE DE REPTATION (*)}

\author{
J. L. PORTESEIL et R. VERGNE \\ Laboratoire de Magnétisme du C. N. R. S., 166 X, 38042 Grenoble Cedex, France
}

(Reçu le 15 décembre 1976, accepté le 5 mai 1977)

\begin{abstract}
Résumé. - On sait depuis longtemps que, lorsqu'on soumet un matériau ferromagnétique à des alternances de champ dissymétriques, les cycles d'hystérésis se déplacent lentement vers les aimantations croissantes (reptation). Pour décrire ce phénomène, L. Néel a proposé un modèle formel faisant intervenir la notion de champ fictif. Dans ce travail, nous essayons de préciser les origines physiques de la reptation. Contrairement aux auteurs précédents qui se limitaient à une seule direction de l'espace, nous étudions le comportement directionnel sur des polycristaux ayant un ou plusieurs axes de facile aimantation( fer, cobalt cubique et hexagonal). La reptation disparaît dans les matériaux uniaxes. Nous suggérons qu'elle est due à des modifications de la structure en domaines rendues possibles par le choix entre plusieurs axes de facile aimantation dans chaque cristallite. L'agitation thermique déclenche des passages aléatoires entre de nombreuses configurations d'énergies voisines. Nous montrons que la structure en domaines s'adapte progressivement à un processus d'aimantation donné et évolue vers un état de plus en plus ordonné.
\end{abstract}

Abstract. - When a ferromagnet is cycled dissymmetrically with respect to $H=0$, the cycles exhibit a slow positive drift (creep or reptation). A formal model involving a fictitious creep field $h_{\mathrm{r}}$ was first presented by L. Néel. In this paper, we investigate the physical origin of this phenomenon. Whereas the previous authors applied all the magnetic fields along one direction, we study the multidirectional behaviour of polycrystals with one or several easy axes (iron, cubic and hexagonal cobalt). The creep disappears in uniaxial materials. We suggest that this phenomenon arises from topological changes in the domain structure related to a possible choice between several easy axes in every crystallite. The thermal fluctuations trigger random jumps between a large number of possible configurations, the energies of which are almost equal. We show that the domain structure adapts itself to a given magnetization process and slowly evolves towards a non-isotropic, ordered state.

1. Introduction. - Bien que l'on connaisse et utilise depuis fort longtemps les matériaux ferromagnétiques, on est loin d'avoir élucidé tous les aspects de leur comportement. L'action d'un champ magnétique sur ces substances se traduit par l'existence d'une courbe de première aimantation et d'un cycle d'hystérésis. D'un point de vue technologique, la connaissance de ces caractéristiques essentielles suffit dans la plupart des cas. Une étude plus fine révèle cependant tout un ensemble de phénomènes considérés le plus souvent comme secondaires. On constate alors que l'aimantation dépend entre autres, du temps, des chocs et contraintes auxquels est soumis l'échantillon, du nombre de cycles qu'on lui fait décrire, de la direction des divers champs appliqués, etc... De tels effets ont été décelés très tôt, mais leur étude approfondie a été

${ }^{*}$ ) Ce travail constitue une partie de la thèse de J. L. Porteseil [1]. délaissée au profit d'autres aspects du magnétisme jugés plus fondamentaux. Ce désintérêt provient en grande partie des difficultés inhérentes à la nature des matériaux ferromagnétiques courants. Ceux-ci possèdent en effet des structures en domaines extrêmement complexes dont le comportement microscopique échappe totalement au calcul.

Les progrès de la cristallogenèse et des techniques de mesure des flux magnétiques permettent aujourd'hui d'étudier des échantillons dont les structures en domaines sont au contraire les plus simples possibles. On retrouve alors des phénomènes identiques, parfois même bien plus accentués que dans les échantillons habituels [1, 2, 3]. Il faut donc abandonner le point de vue d'après lequel ces phénomènes sont marginaux et dus uniquement à des effets statistiques dans les polycristaux. C'est pourquoi, nous avons repris l'étude de certains aspects de l'hystérésis en nous attachant aux caractères fondamentaux des mécanismes d'aiman- 
tation et en essayant de nous abstraire des caractéristiques particulières des substances étudiées. Le présent travail concerne une manifestation de l'hystérésis ferromagnétique connue sous le nom de reptation des cycles de champ.

Ewing [4] observa en 1885 que des cycles successifs dissymétriques par rapport à $H=0$ ne se superposaient pas exactement les uns aux autres et se déplaçaient lentement vers la région des aimantations croissantes (Fig. 1). Bouasse et Berthier [5] rencontrèrent

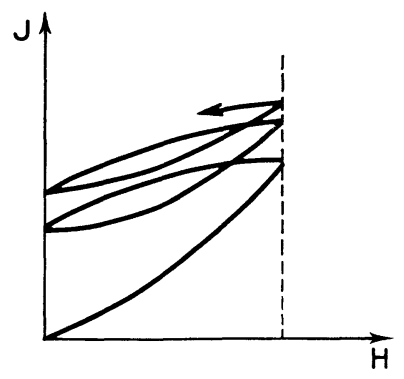

FIG. 1. - Reptation des cycles de champs dissymétriques. [Creep of dissymetric hysteresis loops.]

un effet analogue sur un matériau soumis à des cycles de torsion et lui donnèrent le nom de reptation. Depuis, d'autres auteurs ont étudié certains aspects de ce phénomène [6,7]. Nguyen Van Dang [8] en a donné une description expérimentale détaillée dans des matériaux très divers (fer, différents aciers, ferro-nickel, ferro-cobalt, ferrites, aimants permanents).

2. Le modèle de Néel. — L. Néel [9] a proposé une interprétation théorique de la reptation. Dans un matériau de structure en domaines complexe, il existe de nombreuses structures possibles d'énergies voisines. L. Néel suppose qu'à une même valeur macroscopique de l'aimantation $J$ peuvent correspondre plusieurs distributions de domaines élémentaires. De ce fait, il admet que la configuration microscopique de l'échantillon peut se modifier partiellement et aléatoirement d'un cycle à l'autre. Il en résulte à chaque cycle de nouvelles valeurs des interactions entre domaines et des champs de dispersion dans le matériau. Certains éléments de matière voient alors leur aimantation croître irréversiblement lorsque la situation locale est favorable. On peut décomposer cet accroissement en deux parties dont l'une est acquise définitivement, alors que l'autre sera effacée au cours des cycles ultérieurs.

2.1 SUSCEPTIBILITÉS DIFFÉRENTIELLES RÉVERSIBLE ET IRRÉVERSIBLE. - Avant de poursuivre l'exposé du modèle de $L$. Néel, il est nécessaire de rappeler certaines définitions concernant les propriétés d'un corps ferromagnétique au voisinage d'un point $\mathrm{A}\left(H_{\mathrm{A}}, J_{\mathrm{A}}\right)$ de sa courbe d'aimantation. Supposons que l'état d'aimantation $J_{\mathrm{A}}$ ait été atteint par valeurs croissantes du champ magnétique. Si l'on applique à partir de $H_{\mathrm{A}}$ un champ additionnel $\delta H$ d'amplitude faible devant celle de $H_{\mathrm{A}}$ (Fig. 2), il en résulte un petit accroissement d'aimantation

$$
\delta J=J\left(H_{\mathrm{A}}+\delta H\right)-J\left(H_{\mathrm{A}}\right) .
$$

$\delta J$ n'a pas la même valeur selon que la petite variation de champ est positive ou négative et vaut dans chacun de ces cas [10] :

$$
\left.\begin{array}{l}
J\left(H_{\mathrm{A}}+\delta H\right)-J\left(H_{\mathrm{A}}\right)=\left(S_{\mathrm{r}}+S_{\mathrm{i}}\right) \delta H \\
J\left(H_{\mathrm{A}}-\delta H\right)-J\left(H_{\mathrm{A}}\right)=-S_{\mathrm{r}} \delta H
\end{array}\right\} .
$$

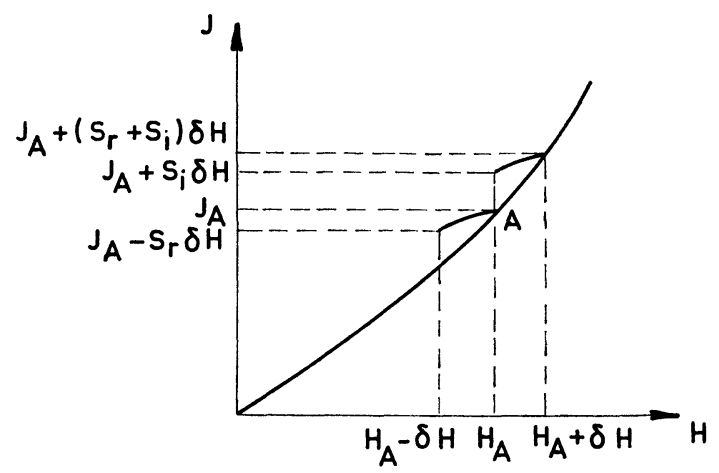

Fig. 2. - Définition des susceptibilités réversible et irréversible $S_{\mathrm{r}}$ et $S_{\mathrm{i}}$.

[Definition of the reversible and irreversible susceptibilities $S_{\mathbf{r}}$ and $S_{1 .]}$

On définit ainsi les susceptibilités différentielles réversible et irréversible $S_{\mathrm{r}}$ et $S_{\mathrm{i}}$. Après retour du champ à la valeur $H_{\mathrm{A}}$, le terme irréversible $S_{\mathrm{i}} \delta H$ est acquis et gardera la même valeur tant que les variations ultérieures du champ resteront inférieures à $\delta H$. Si, au contraire, le champ additionnel prend la valeur $\delta H^{\prime}>\delta H$, le terme irréversible devient $S_{\mathrm{i}} \delta H^{\prime}$.

Les suceptibilités dépendent non seulement des valeurs actuelles de $H_{\mathrm{A}}$ et $J_{\mathrm{A}}$ mais aussi de l'histoire magnétique de l'échantillon. Dans le domaine des champs faibles $\left(\sim H_{c} / 10\right)$, elles obéissent à des lois simples connues sous le nom de lois de Rayleigh :

- La courbe de première aimantation a pour expression :

$$
J=a H+b H^{2} .
$$

- Lorsque le champ a atteint une valeur $H_{1}$ par valeurs décroissantes et l'aimantation une valeur $J_{1}$ si on fait croître le champ à partir de $H_{1}$, la variation d'aimantation correspondante est :

$$
J-J_{1}=a\left(H-H_{1}\right)+\frac{b}{2}\left(H-H_{1}\right)^{2} .
$$

Lorsque le champ a atteint la valeur $H_{2}$ en croissant, si on le fait décroître, la variation d'aimantation vaut alors :

$$
J-J_{2}=a\left(H-H_{2}\right)-\frac{b}{2}\left(H-H_{2}\right)^{2} .
$$


Dans ces conditions, les susceptibilités réversible et irréversible s'expriment de façon simple. $S_{\mathrm{r}}$ est constamment égale à $a$, tandis que $S_{\mathrm{i}}$ vaut $2 b H_{\mathrm{A}}$ ou $b\left(H_{\mathrm{A}}-H\right)$ suivant que l'on étudie la courbe de première aimantation ou la branche descendante du cycle d'hystérésis.

Nous n'avons considéré jusqu'à présent que l'accroissement d'aimantation qui résulte de la seule application d'un champ additionnel. Nous nous proposons maintenant d'exprimer l'aimantation irréversible qui reste acquise lorsque, après application du champ additionnel, on donne au champ la valeur $H_{\mathrm{B}}<H_{\mathrm{A}}$ avant de rétablir le champ $H_{\mathrm{A}}$ (Fig. $3 a$ ). On écrit symboliquement :

$\delta J=J\left(H_{\mathrm{A}}+\delta H, H_{\mathrm{B}}, H_{\mathrm{A}}\right)-J\left(H_{\mathrm{A}}, H_{\mathrm{B}}, H_{\mathrm{A}}\right)=S_{\mathrm{AB}} \delta H$.

De même, la variation d'aimantation au point $\mathbf{B}$ due à l'application de $\delta H$ au point $\mathrm{A}$ (Fig. $3 b$ ) s'écrit :

$\delta J=J\left(H_{\mathrm{A}}+\delta H, H_{\mathrm{B}}\right)-J\left(H_{\mathrm{A}}, H_{\mathrm{B}}\right)=S_{\mathrm{BA}} \delta H$.

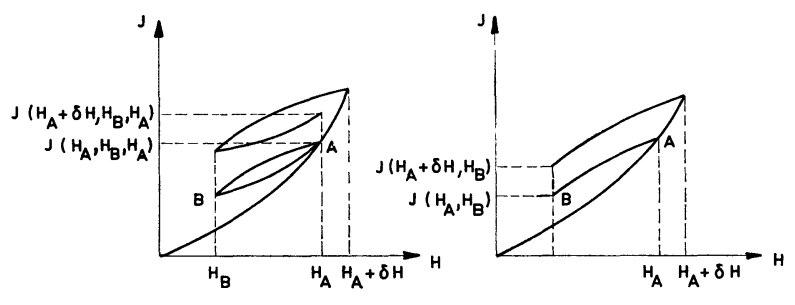

(a)

(b)

Fig. 3. - Définition des susceptibilités irréversibles composées $S_{\mathrm{AB}}$ et $S_{\mathrm{BA}}$.

[Definition of the compound irreversible susceptibilities $S_{\mathrm{AB}}$ and $S_{\text {BA. }}$ ]

On définit ainsi les susceptibilités composées $S_{\mathrm{AB}}$ et $S_{\mathrm{BA}}$ qui dépendent de $H_{\mathrm{A}}$, de $H_{\mathrm{B}}$ et de l'histoire magnétique de l'échantillon. Dans le domaine de Rayleigh, $S_{\mathrm{AB}}$ et $S_{\mathrm{BA}}$ sont toutes deux égales à la quantité $b\left(H_{\mathrm{A}}+H_{\mathrm{B}}\right)$. Si le cycle est symétrique par rapport à $H=0\left(H_{\mathrm{B}}=-H_{\mathrm{A}}\right)$, les susceptibilités composées sont nulles. On admet généralement que l'égalité $S_{\mathrm{AB}}=S_{\mathrm{BA}}$ subsiste hors du domaine de Rayleigh. Nous reviendrons en détail sur ce point.

2.2 Le CHAMP FICTIF DE REPTATION. - L. Néel propose de décrire la reptation des cycles par l'action d'un petit champ magnétique de caractère aléatoire venant se superposer au champ appliqué $H_{\mathrm{A}}$. Ce champ représente la partie fluctuante des interactions entre domaines élémentaires. En un point donné du matériau, il conserve la même valeur tant que le champ appliqué reste constant. Par contre, il fluctue dans l'espace de façon aléatoire et se caractérise par une valeur moyenne nulle et une valeur quadratique moyenne égale à $h_{\mathrm{r}}^{2}$.

Appelons $\delta H_{n}$ la valeur de ce champ à la $n^{\mathrm{e}}$ application de $H_{\mathrm{A}}$ et en un point donné de l'échantillon.
On observe en ce point un accroissement d'aimantation $\delta J_{n}$ qui est la somme de deux termes :

- un terme réversible égal à $S_{\mathrm{r}} \delta H_{n}$;

- un terme irréversible qui correspond à la plus grande valeur du champ aléatoire ayant été atteinte en ce point dans le passé. Soit $\delta H_{m}$ cette valeur maximale $(m \leqslant n)$. Puisque le champ magnétisant a décrit des cycles entre $H_{\mathrm{A}}$ et $H_{\mathrm{B}}$, il subsiste au point $\mathrm{A}$ un terme irréversible égal à $S_{\mathrm{AB}} \delta H_{m}$.

La structure en domaines d'un matériau ferromagnétique ordinaire étant extrêmement complexe, on observe à notre échelle la moyenne spatiale de la quantité $S_{\mathrm{r}} \delta H_{n}+S_{\mathrm{AB}} \delta H_{m} \cdot\left\langle\delta H_{n}\right\rangle$ est nul par hypothèse. Par contre, $<\delta H_{m}>$ est une fonction de $n$ croissante et positive puisque à chaque nouveau tirage au sort, on peut espérer obtenir en n'importe quel point, une valeur du champ aléatoire supérieure à toutes celles qu'il a prises dans le passé. Il est commode d'exprimer la valeur de $\left\langle\delta H_{m}\right\rangle$ à la $n^{\mathrm{e}}$ application de $H_{\mathrm{A}}$ en fonction de la valeur quadratique moyenne $h_{\mathrm{r}}^{2}$ du champ aléatoire sous la forme :

$$
<\delta H_{m}>=x_{n} h_{\mathrm{r}}
$$

$x_{n}$ est un facteur numérique dont les variations en fonction de $n$ dépendent de la loi de probabilité à laquelle obéit le champ aléatoire. $x_{1}$ est toujours égal à 0 puisque la valeur $n=1$ correspond à la première application de $H_{\mathrm{A}}$ sans retour au champ $H_{\mathrm{B}}$. C'est pourquoi il est plus commode de porter les valeurs de $x_{n}$ en fonction de $n-1$. L. Néel a calculé les variations de $x_{n}$ dans le cas d'une loi de Gauss. Le tableau I donne les premières valeurs de $x_{n}$ :

\section{TABleau I}

Variations de la quantité $x_{n}$ pendant les premiers cycles, calculées dans le cas d'une loi de Gauss

[Variation of the quantity $x_{n}$ during the first few cycles, calculated in the case of a Gauss law]

$\begin{array}{llllll}n-1 & 0 & 1 & 2 & 3 & 4 \\ x_{n} & 0 & 0,5642 & 0,8463 & 1,0293 & 1,1629\end{array}$

Lorsque $n$ est grand, $x_{n}$ croît comme $(\log n)^{1 / 2}$.

3. Méthode de mesure. - Les variations irréversibles d'aimantation dues au traînage de fluctuations thermiques se superposent inévitablement aux accroissements d'aimantation qui nous intéressent. Si l'on veut mesurer la reptation avec précision, il faut donc séparer les effets des paramètres temps et nombre de cycles. Il ressort d'une étude de L. Néel [10] qu'une telle séparation est possible si on utilise une méthode indirecte qui consiste à mesurer la susceptibilité différentielle irréversible à l'extrémité supérieure des cycles (Fig. 4). Après les $n$ applications successives du champ $H_{\mathrm{A}}$, on mesure l'aimantation $J$ au point A. On applique alors un champ additionnel $\delta H$, puis on le supprime 


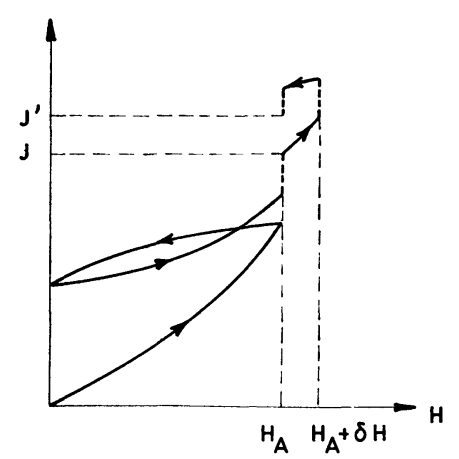

Fig. 4. - Méthode de mesure indirecte permettant de séparer le traînage et la reptation. Les pointillés représentent les accroissements irréversibles d'aimantation, dus au traînage de fluctuations.

[Indirect method allowing separation of the irreversible increase of magnetization due to the fluctuation after-effect.]

et on mesure la nouvelle valeur $J^{\prime}$ de l'aimantation. L'accroissement d'aimantation irréversible

$$
\delta J_{n-1}=J^{\prime}-J
$$

reflète la compétition des deux champs aléatoires de traînage et de reptation.

$\mathrm{La}$ séquence correspondante d'application des champs est schématisée par la figure 5 où l'on a supposé

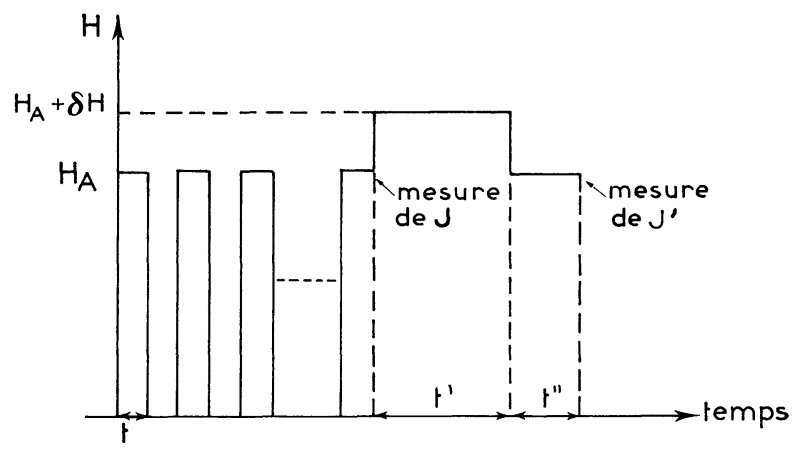

Fig. 5. - Séquence d'application des champs principal et additionnel.

[Time schedule of the main and additional magnetic fields.]

$H_{\mathrm{B}}=0$ pour simplifier. Il est commode de maintenir le champ principal $H_{\mathrm{A}}$ pendant le même temps $t$ à chacun des $n$ cycles. Après avoir mesuré $J$, on applique le champ additionnel $\delta H$ pendant un temps $t^{\prime}$. On supprime alors $\delta H$ et on mesure $J^{\prime}$ après qu'un autre temps $t^{\prime \prime}$ se soit écoulé. $\delta J_{n-1}$ est une fonction assez complexe des temps $t, t^{\prime}, t^{\prime \prime}[10]$. Toutefois, son expression se simplifie notablement si on choisit $t=t^{\prime}=t^{\prime \prime}$. Nous avons respecté systématiquement cette condition.

L'expression de $\delta J_{n-1}$ n'est pas la même selon les amplitudes respectives des champs fictifs de traînage et de reptation. L. Néel a montré [10] que si l'un des deux champs est assez nettement supérieur à l'autre (il suffit d'un facteur 2), il l'inhibe complètement. Si l'on prend de plus la précaution d'appliquer un champ $\delta H$ au moins égal à quelques fois la constante de traînage $S$ [11], l'accroissement $\delta J_{n-1}$ a pour expression dans le cas où le traînage est prépondérant :

$\delta J_{n-1}=J^{\prime}-J=S_{\mathrm{i}} \delta H-S_{\mathrm{AB}} S \log n-S_{\mathrm{AB}} \frac{h_{\mathrm{r}}^{2}}{2 S}$.

Le terme $S_{\mathrm{AB}} S \log n$ représente l'accroissement irréversible d'aimantation dû à l'action cumulée du traînage au cours des $n$ cycles. On le nomme reptation thermique. Le terme $S_{\mathrm{AB}} \frac{h_{\mathrm{r}}^{2}}{2 S}$ est petit et généralement négligeable : les effets de la reftation proprement dite ont disparu.

$\mathrm{Si}$ au contraire, la reptation l'emporte, $\delta J_{n-1}$ vaut :

$$
\delta J_{n-1}=J^{\prime}-J=S_{\mathrm{i}} \delta H-S_{\mathrm{AB}} x_{n} h_{\mathrm{r}} .
$$

Si les champs de traînage et de reptation sont du même ordre de grandeur, ou si l'on n'a pas pris la précaution d'appliquer un champ additionnel assez nettement supérieur à la constante de traînage $S$, le calcul rigoureux de $\delta J_{n-1}$ devient inextricable.

Compte tenu des expressions (8) et (9), nous avons adopté la méthode de mesure suivante. Après désaimantation, on applique à l'échantillon le programme de champs schématisé par la figure 5 et on mesure la quantité $\delta J_{n-1}$. On a d'autre part accès à la quantité $\delta J_{0}=S_{\mathrm{i}} \delta H$ par une mesure ordinaire de susceptibilité irréversible. Ici encore, un minutage approprié permet de rendre reproductible l'influence du traînage [11]. La différence $\Delta=\delta J_{0}-\delta J_{n-1}$ est égale à :

$$
\Delta=S_{\mathrm{AB}} S \log n+S_{\mathrm{AB}} \frac{h_{\mathrm{r}}^{2}}{2 S}
$$

si le traînage est prépondérant, et :

$$
\Delta=S_{\mathrm{AB}} x_{n} h_{\mathrm{r}}
$$

dans le cas contraire.

Ayant mesuré par ailleurs la constante de traînage $S$, on calcule le terme de reptation thermique $S \log n$. On attribue à un traînage prépondérant toutes les valeurs expérimentales de $\Delta / S_{\mathrm{AB}}$ voisines de cette quantité. Si au contraire $\Delta / S_{\mathrm{AB}}$ est nettement supérieur à $S \log n$, on l'identifie au terme de reptation proprement dit $x_{n} h_{\mathrm{r}}(11)$.

\section{Paramètres physiques dont dépend le phénomène} de reptation. - Les études précédentes portaient sur des substances variées mais ayant toutes une structure cubique. De tels matériaux ont plusieurs directions de facile aimantation qui peuvent être les axes cristallographiques du type $<100\rangle$ (fer) ou $<111\rangle$ (nickel, cobalt en phase cubique) suivant que la constante d'anisotropie $K_{1}$ est positive ou négative. Nous avons repris l'étude de la reptation sur de tels matériaux, mais également sur des substances magnétiquement uniaxes (cobalt hexagonal). 
Si l'on met à part quelques expériences de reptation transversale [13], les auteurs précédents appliquaient tous les champs magnétiques dans une seule direction de l'espace. C'est restreindre inutilement la généralité du problème. Nous avons d'ailleurs montré $[12,14]$ que des phénomènes apparemment complexes tels que l'anisotropie de désaimantation s'éclaircissent considérablement si on étudie leurs aspects pluridirectionnels. C'est pourquoi nous avons choisi d'appliquer les champs principal et additionnel dans toutes les directions d'un plan. Dans la suite de l'exposé, nous appellerons $\psi$ l'angle des vecteurs $\mathbf{H}_{\mathrm{A}}$ et $\delta \mathbf{H}$ (Fig. 6).

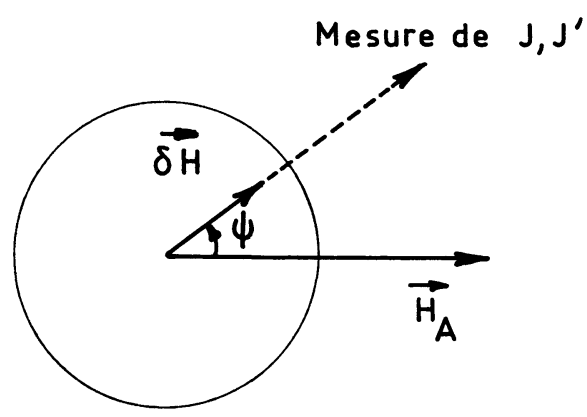

FIG. 6. - Application des champs principal et additionnel suivant différentes directions d'un plan.

[Applying the main and additional fields along various directions in a plane.]

Les variations de la quantité $x_{n}$ sont assez rapides pour les faibles valeurs de $n$ et beaucoup plus lentes ensuite. Nguyen Van Dang [8] n'a d'ailleurs pas observé d'écart à la loi asymptotique en $(\log n)^{1 / 2}$ sur des nombres de cycles allant jusqu'à $10^{6}$. C'est pourquoi nous nous sommes limités en pratique à $n-1=10$. Pour ces faibles valeurs du paramètre nombre de cycles apparaissent au contraire des phénomènes intéressants sur lesquels nous reviendrons en détail.

Une autre variable physique essentielle est l'amplitude des cycles. L. Néel suggère [10] que les remaniements aléatoires de la structure en domaines sont d'autant plus importants que le matériau subit de grandes variations d'aimantation. On sait d'autre part [8] que la reptation est la plus forte lorsque l'amplitude des cycles de champ $H_{\mathrm{A}}-H_{\mathrm{B}}$ est de l'ordre du champ coercitif $H_{\mathrm{c}}$. Au contraire, le traînage tend à supplanter la reptation dans la région des champs faibles $\left(\sim 0,3 H_{\mathrm{c}}\right)$. Nous avons fait systématiquement $H_{\mathrm{B}}=0$ pour simplifier et nous avons exploré la gamme de champs comprise entre $H_{\mathrm{c}} / 4$ et $H_{\mathrm{c}} / 2$ environ. Dans cette région, les mécanismes d'aimantation sont dus essentiellement à des déplacements de parois et suivent de près les lois de Rayleigh.

5. Les échantillons et le dispositif expérimental. Pour mettre en évidence et mesurer les phénomènes de reptation, le choix de la substance n'est pas critique. Il suffit de disposer d'un polycristal sans orientations préférentielles notables dans le plan où l'on applique les champs magnétiques. D'autre part, les domaines doivent être nombreux et répartis de façon complexe pour que l'on puisse exprimer les variations d'aimantation par des intégrales sur des distributions continues de parois. L'anisotropie magnétocristalline doit également être assez forte pour que les déplacements de parois soient prépondérants dans la gamme de champs qui nous intéresse $\left(\sim H_{\mathrm{c}} / 2\right)$. Lorsque ces diverses conditions sont remplies, le calcul des variations d'aimantation devient possible.

Nous avons utilisé d'une part un échantillon d'acier déjà bien connu car il nous avait servi auparavant à étudier l'anisotropie de désaimantation. Sa composition en poids est : $\mathrm{Fe}=93,4 \% ; \mathrm{C}=0,3 \%$; $\mathrm{Ni}=4,3 \% ; \mathrm{Cr}=1,5 \% ; \mathrm{Mo}=0,5 \%$. Les trois axes de facile aimantation de chaque cristallite sont dirigés suivant les directions quaternaires du type $<100>$.

Un autre échantillon est composé de cobalt à $99,9 \%$, le nickel et le fer constituant la majeure partie des impuretés. Il a été recuit vers $600^{\circ} \mathrm{C}$ de manière à rendre prépondérante la phase cubique du cobalt. Les axes de facile aimantation sont cette fois du type $<111>$.

Enfin, un autre échantillon de cobalt de même pureté a été soumis à un écrouissage et à un traitement thermique vers $400^{\circ} \mathrm{C}$. Le matériau ainsi obtenu est presque entièrement en phase hexagonale et par conséquent uniaxe du point de vue magnétique.

Pour envisager la reptation dans toute sa généralité, il faudrait appliquer les champs magnétiques dans toutes les directions de l'espace. Cela imposerait d'étudier des échantillons sphériques dont le coefficient de champ démagnétisant serait prohibitif compte tenu des limites de notre dispositif expérimental. D'autre part, on ne sait pas obtenir en pratique un état désaimanté isotrope suivant toutes les directions de l'espace, si ce n'est en réchauffant l'échantillon au-dessus de la température de Curie et en le laissant refroidir en champ nul (désaimantation thermique). C'est pourquoi nous nous sommes bornés à étudier un problème plan. Les trois échantillons ont la forme de disques minces: $\varnothing=60 \mathrm{~mm}, e=1,2 \mathrm{~mm}$. En les assimilant à des ellipsoïdes aplatis, on obtient pour le coefficient de champ démagnétisant la valeur $N=0,20$. Un ensemble de bobines [15] permet d'appliquer les champs magnétiques $H_{\mathrm{A}}$ et $\delta H$ dans toutes les directions du plan du disque et de compenser le champ terrestre. Ces bobines servent également à la désaimantation alternative de l'échantillon. Nous avons pris pour l'état désaimanté de référence, l'état isotrope obtenu en faisant tourner le disque à vitesse constante dans un champ alternatif d'amplitude lentement décroissante [15].

Nous avons mesuré les variations d'aimantation à l'aide d'un fluxmètre électronique qui a déjà été décrit en détail [16]. A cet appareil est associée une mutuelle inductance réglable, permettant de compenser les variations réversibles de flux qui constituent la majeure partie des phénomènes (de 70 à $97,5 \%$ de la variation 
totale de flux suivant les cas). Nous pouvons ainsi mesurer avec précision de très faibles variations d'aimantation irréversible.

6. Les résultats expérimentaux. - 6.1 Résultats RELATIFS A L'ÉCHANTILlON D'ACIER. - Des mesures préliminaires nous ont permis de déterminer son champ coercitif $H_{\mathrm{c}}=14,7 \mathrm{Oe}$ et sa constante de traînage à la température ambiante $S=15,0 \mathrm{mOe}$.

6.1.1 Mesure des quantités $\delta J_{n-1}$. La figure 7 reproduit un enregistrement typique des variations d'aimantation qui se produisent à partir de la $n^{\mathrm{e}}$ application du champ $H_{\mathrm{A}}$. Les divers paramètres ont les valeurs suivantes :

$$
\begin{aligned}
& H_{\mathrm{A}}=0,7 \mathrm{Oe} \quad \delta H=0,148 \mathrm{Oe} \quad n-1=1 \\
& \psi=0^{\circ}\left(H_{\mathrm{A}} \text { et } \delta H \text { colinéaires }\right) .
\end{aligned}
$$

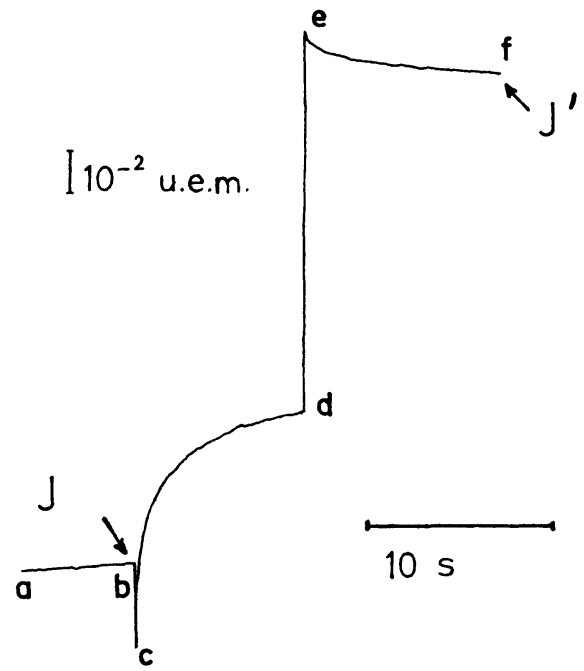

FIG. 7. - Variations d'aimantation enregistrées au cours d'une mesure de la quantité $\delta J_{n-1}$. ab : traînage de l'aimantation sous champ constant $H_{\mathrm{A}} ;$ bc : régime transitoire dû à l'application du champ additionnel $\delta H$ (la légère surcompensation des termes réversibles est responsable de la diminution du flux); cd : traînage sous champ constant $H_{\mathrm{A}}+\delta H$; de : régime transitoire dû à la suppression du champ additionnel $\delta H$; ef : traînage après retour du champ à la valeur $H_{\mathrm{A}}$.

[Changes of magnetization recorded during the measurement of the quantity $\delta J_{n-1}$. ab : after-effect in a constant field $H_{\mathrm{A}}$; bc : transient change of magnetic flux due to the additional field $\delta H$ (the slight overcompensation of the reversible magnetization is responsible for the decrease of the magnetic flux); cd : aftereffect in a constant field $H_{\mathrm{A}}+\delta H$; de : transient change of flux due to the suppression of $\delta H$; ef : after-effect when the applied field has returned to the value $H_{\mathrm{A}}$.]

Les trois parties de l'enregistrement de gauche à droite correspondent respectivement à la fin du $n^{\mathrm{e}}$ cycle, à l'application du champ additionnel et à l'évolution après coupure de celui-ci. L'ordonnée de la partie centrale subit un décalage arbitraire dû à la compensation des termes purement réversibles par la mutuelle réglable. Cela n'est pas gênant puisqu'on ne s'intéresse qu'à la différence $J^{\prime}-J$. On voit clairement sur cette figure l'évolution de l'aimantation sous l'effet du traînage au cours des diverses phases de la mesure, et on constate l'importance essentielle d'un minutage correct sans lequel on peut commettre des erreurs très importantes.

6.1.2 Forme des courbes $\Delta(\delta H)$. - La figure 8 montre comment varie la quantité $\Delta=\delta J_{0}-\delta J_{n-1}$

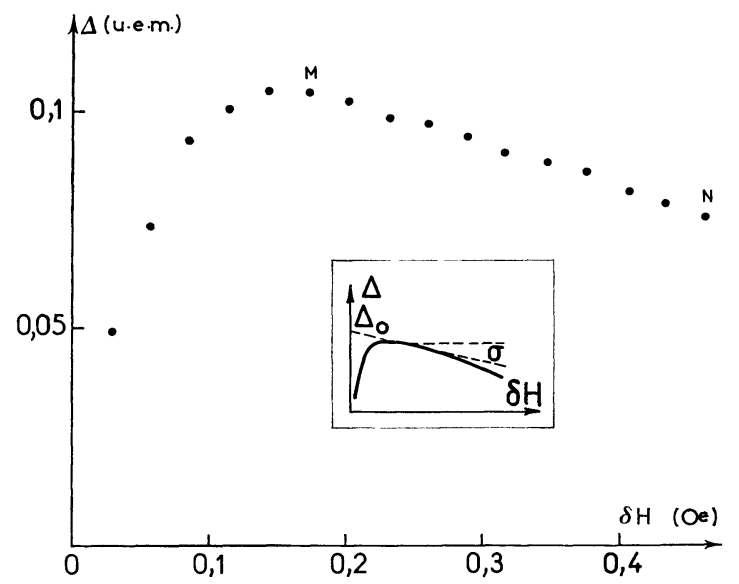

Fig. 8. - Variation de la quantité $\Delta$ en fonction de l'amplitude du champ additionnel $\delta H$; définition des paramètres $\Delta_{0}$ et $\sigma$. [Variation of the quantity $\Delta$ plotted versus the magnitude of the additional field $\delta H$.]

en fonction de l'amplitude du champ additionnel. Les conditions expérimentales sont $H_{\mathrm{A}}=7,57$ Oe, $n-1=10, \psi=0^{\circ}$. La décroissance rapide de $\Delta$ pour les faibles valeurs du champ additionnel est due au fait que $\delta H$ n'est pas assez grand vis-à-vis de la constante de traînage $S$. Nous ne considérerons les valeurs de $\Delta$ comme correctes qu'à partir de $\delta H \sim 120 \mathrm{mOe}$, soit $\delta H / S \sim 8$.

On constate pour les grandes valeurs de $\delta H$ que la quantité $\Delta$ décroît de façon sensiblement linéaire, en contradiction avec les expressions (10) et (11), d'après lesquelles $\Delta$ doit être indépendant de $\delta H$. Ce résultat est général et la pente de la partie rectiligne $\mathrm{MN}$ dépend de $H_{\mathrm{A}}$, de $n-1$ et de $\psi$.

On peut montrer que la variation $\mathrm{MN}$ de la fonction $\Delta(\delta H)$ est parabolique si les champs mis en jeu n'excèdent pas la limite du domaine de Rayleigh :

$$
\Delta=\Delta_{0}+\sigma \delta H+\alpha \delta H^{2} .
$$

Le terme $\alpha$ étant petit, on assimilera $\mathrm{MN}$ à une droite de pente $\sigma$ et d'ordonnée à l'origine $\Delta_{0}$. Cependant, pour déterminer $\sigma$ et $\Delta_{0}$, nous avons ajusté les résultats expérimentaux sur une loi parabolique par une méthode de moindres carrés. On trouve ainsi pour la courbe de la figure 8 :

$$
\Delta_{0}=0,1352 \text { u. e. m. ; } \sigma=0,085 \text { u. e. m. } \mathrm{Oe}^{-1} \text {. }
$$

La grandeur $\sigma$ a les dimensions d'une susceptibilité et représente dans ce cas environ $4,5 \%$ de la susceptibilité irréversible ordinaire :

$$
S_{\mathrm{i}}=1,87 \text { u. e. m. } \mathrm{Oe}^{-1} \text {. }
$$




\section{TABLEAU II}

Le champ de reptation $h_{\mathrm{r}}$ en fonction de l'amplitude du champ principal (échantillon d'acier) [The creep field $h_{\mathrm{r}}$ as a function of the magnitude of the main field (steel sample)]

$\begin{array}{lcccccccc}\boldsymbol{H}_{\mathrm{A}}(\mathrm{Oe}) & 2,64 & 3,33 & 3,84 & 4,74 & 5,67 & 6,61 & 7,15 & 7,57 \\ H_{\mathrm{A}} / H_{\mathrm{c}} & 0,180 & 0,227 & 0,261 & 0,322 & 0,386 & 0,450 & 0,486 & 0,515 \\ \Delta_{\mathrm{o}} / \boldsymbol{S}_{\mathrm{AB}}(\mathrm{mOe}) & 38,1 & 37,8 & 40,1 & 56,8 & 80,6 & 103,1 & 126,2 & 145,9 \\ h_{\mathrm{r}}(\mathrm{mOe}) & (24,0) & (23,8) & (25,3) & (35,8) & 50,8 & 65,0 & 79,6 & 92,0\end{array}$

6.1.3 Influence du paramètre $H_{\mathrm{A}}$. - L'étude de la reptation implique de connaître la susceptibilité irréversible composée $S_{\mathrm{AB}}$ pour chaque valeur du champ principal $H_{\mathrm{A}}$. Nous avons mesuré cette grandeur par application d'un programme de champs qui découle directement de la figure $3 a$. Ici encore un minutage approprié permet d'éliminer l'influence du traînage de fluctuations thermiques.

On indique dans le tableau II, les valeurs mesurées de $\Delta_{0} / S_{\mathrm{AB}}$ pour huit valeurs de $H_{\mathrm{A}}$. Ces quantités sont à comparer avec $S \log n$. Dans toutes ces expériences on a pris : $\psi=0^{\circ} ; n-1=10 ; x_{n}=1,586 ; \log n=2,398$; $S \log n=36,0$ mOe.

On constate, à la lecture de ce tableau, que les quatre colonnes de droite donnent pour $\Delta_{0} / S_{\mathrm{AB}}$ des valeurs nettement supérieures au terme de reptation thermique $S \log n$. On peut donc les attribuer à un phénomène de reptation proprement dite. Les points correspondants sont indiqués par des cercles pleins sur la figure 9 ;

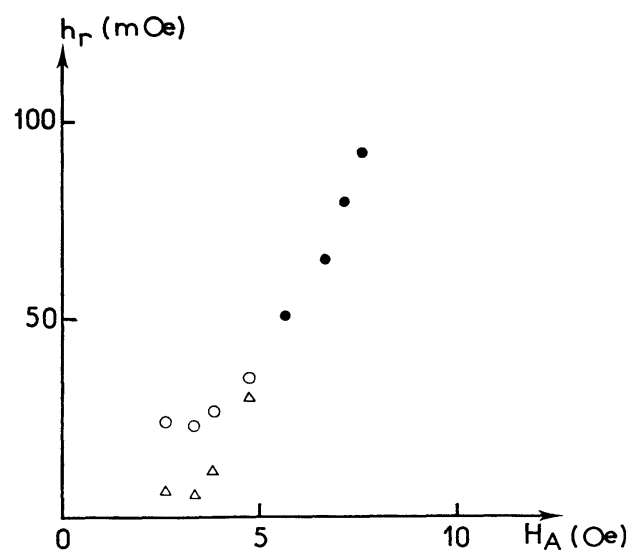

FIG. 9. - Compétition du traînage et de la reptation en fonction de l'amplitude des cycles. Cercles pleins : reptation proprement dite. Cercles vides : reptation thermique.

[Competition between the fluctuation aftereffect and the creep. Full circles : creep properly so-called. Open circles : thermal creep.] on constate que le champ de reptation est une fonction rapidement croissante de $H_{\mathrm{A}}$. Au contraire, les quatre colonnes de gauche indiquent des valeurs de $\Delta_{0} / S_{\mathrm{AB}}$ assez peu différentes de $S \log n=36,0 \mathrm{mOe}$. On donne entre parenthèses les valeurs correspondantes de la quantité $\Delta_{0} / S_{\mathrm{AB}} x_{n}$ que l'on ne peut alors identifier au champ de reptation $h_{\mathrm{r}}$. Les trois premières colonnes correspondent à un phénomène prépondérant de traînage tandis que la quatrième colonne reflète vraisemblablement la compétition d'un traînage et d'une reptation d'amplitudes comparables. On a porté à titre indicatif les points correspondants (cercles vides) sur la figure 9 pour montrer comment la courbe atteint une valeur constante correspondant à la reptation thermique lorsque $H_{\mathrm{A}}$ diminue.

On peut néanmoins essayer d'estimer la valeur de $h_{\mathbf{r}}$ dans le cas où le traînage l'emporte en mettant l'expression (10) sous la forme :

$$
h_{1}^{2}=2 S\left(\frac{\Delta_{0}}{S_{\mathrm{AB}}}-S \log n\right) \text {. }
$$

Les résultats obtenus sont indiqués sur la figure 9 par des triangles.

6.1.4 Influence du nombre de cycles. - Nous avons regroupé dans le tableau III, les valeurs expérimentales des quantités $\Delta_{0} / S_{\mathrm{AB}}$ et $h_{\mathrm{r}}$ pour les valeurs de $n-1$ comprises entre 1 et 10 . Les autres paramètres valent respectivement $H_{\mathrm{A}}=7,57$ Oe et $\psi=0^{\circ}$.

On constate que le champ de reptation ainsi déterminé, au lieu d'être constant, est une fonction croissante du nombre de cycles. Nguyen Van Dang [8] a déjà signalé ce résultat et a montré que le rapport $\Delta_{0} / S_{\mathrm{AB}} x_{n}$ ne devient sensiblement constant qu'à partir de $n=50$ ou 100. Un comportement analogue a été observé par G. Rimet [17] sur des échantillons soumis à des cycles de contraintes mécaniques.

6.1.5 Influence de la variable angulaire $\psi$. - L'état désaimanté initial étant isotrope dans le plan du

\section{TABLEAU III}

Valeurs expérimentales du champ de reptation $h_{\mathrm{r}}$ : dépendance du nombre de cycles

[Experimental values of the creep field $h_{\mathrm{r}}$ : dependence on the number of cycles]

\begin{tabular}{|c|c|c|c|c|c|c|c|c|c|c|}
\hline$n-1$ & 1 & 2 & 3 & 4 & 5 & 6 & 7 & 8 & 9 & 10 \\
\hline$\Delta_{0} / S_{\mathrm{AB}}(\mathrm{mOe})$ & 43,8 & 69,8 & 88,4 & 102,7 & 114,5 & 124,1 & 130,2 & 136,0 & 141,3 & 145 \\
\hline$\Delta_{0} / S_{\mathrm{A} \mathrm{B}} x_{n}$ & 77,7 & 82,5 & 85,9 & 88,3 & 90,4 & 91,8 & 91,5 & 91,6 & 91,9 & $0 ?$ \\
\hline
\end{tabular}


disque, la direction suivant laquelle on applique le champ principal est sans importance. Seul compte l'angle $\psi$ entre les directions des champs principal et additionnel.

De même, la symétrie du problème par rapport à la direction de $\mathbf{H}_{\mathrm{A}}$ impose que les variations angulaires des divers phénomènes soient des fonctions paires de $\psi$. Ces deux conséquences sont effectivement vérifiées dans la limite des erreurs expérimentales. Dès lors, nous nous bornerons aux valeurs positives de $\psi$.

6.1.5.1 Variations de $\Delta_{0}$. - On a tracé sur les figures 10 et 11 les réseaux de courbes représentant les variations de $\Delta_{0}(\psi)$ pour différentes valeurs de $H_{\mathrm{A}}$ d'une part, de $n-1$ d'autre part. On constate que ces fonctions passent au voisinage de $\psi=45^{\circ}$ par des maxima d'autant plus nets que le champ et le nombre de cycles sont plus faibles.

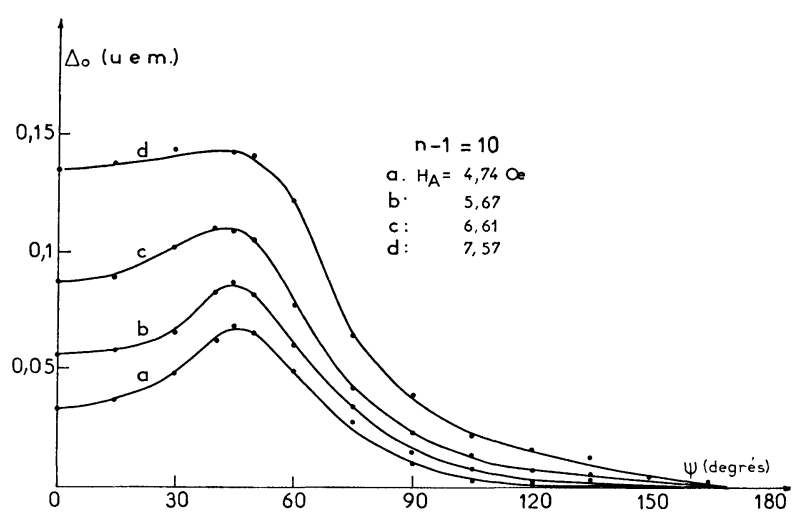

Fig. 10. - Variation angulaire de la quantité $\Delta_{0}(\psi)$ pour différentes valeurs du champ principal.

[Angular variation of $\Delta_{0}(\psi)$ for different values of the main field.]

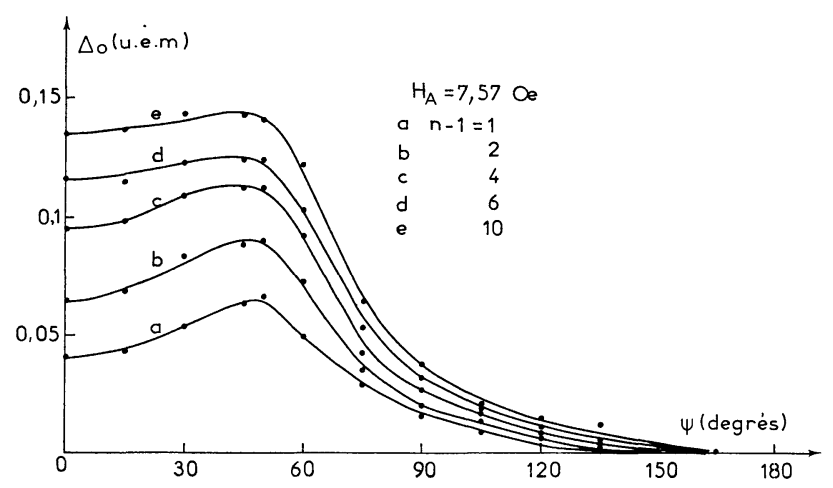

Fig. 11. - Variation angulaire de la quantité $\Delta_{0}(\psi)$ pour différentes valeurs du nombre de cycles.

[Angular variation of $\Delta_{0}(\psi)$ for different numbers of cycles.]

6.1.5.2 Variations de $\sigma$ à l'extrémité A du cycle. Le comportement de $\sigma$ est schématisé par les réseaux de courbes des figures 12 et 13 , où les paramètres sont respectivement $H_{\mathrm{A}}$ et $n-1$.

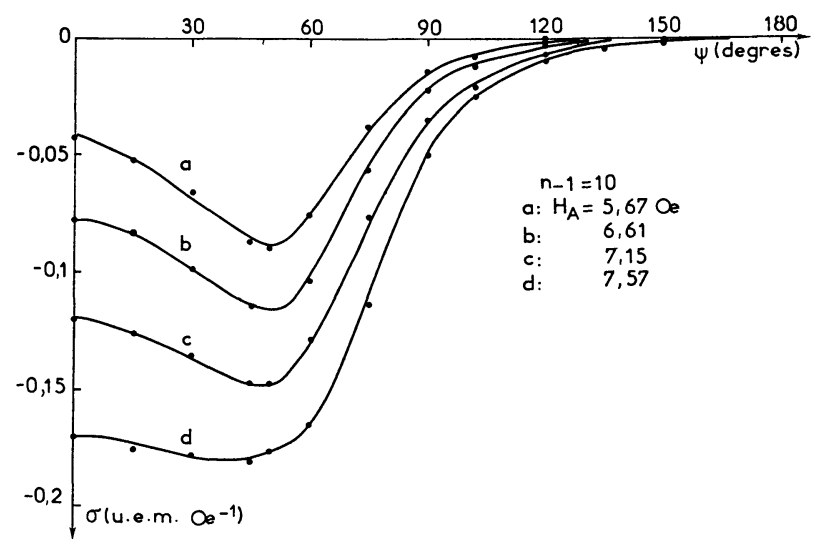

FIG. 12. - Variation angulaire de $\sigma(\psi)$ mesurée à l'extrémité A du cycle pour différentes valeurs du champ principal.

[Angular variation of $\sigma(\psi)$ measured at the upper corner $\mathrm{A}$ of the loop for different values of the main field.]

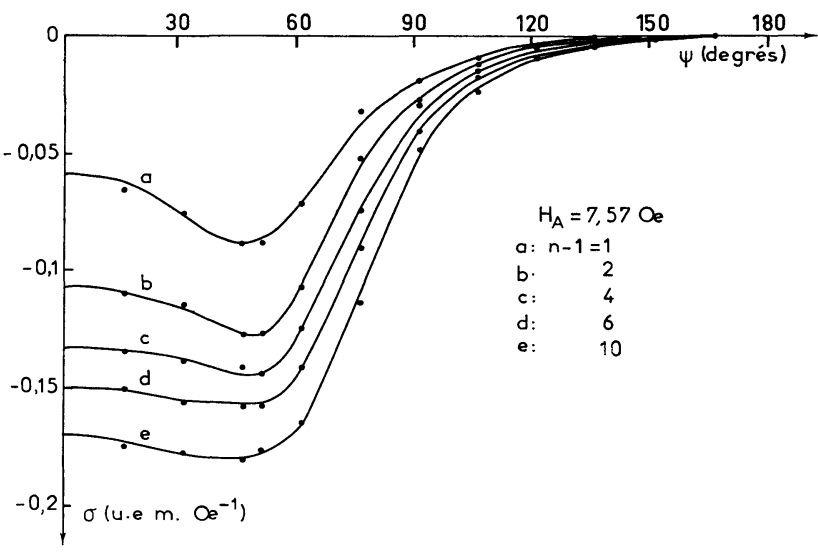

FIG. 13. - Variation angulaire de $\sigma(\psi)$ mesurée à l'extrémité A du cycle pour différentes valeurs du nombre de cycles.

[Angular variation of $\sigma(\psi)$ at the upper corner $\mathbf{A}$ of the loop for different numbers of cycles.]
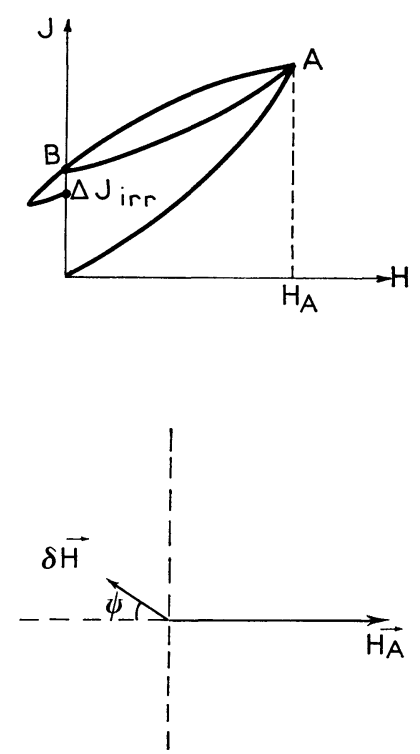

Fig. 14. - Mesure des susceptibilités à l'extrémité $B$ des cycles. [Measurement of the irreversible susceptibility at the lower corner B of the loop.] 
6.1 5.3 Variations de $\sigma$ à l'extrémité B du cycle. Il est également possible de mesurer les susceptibilités irréversibles à l'extrémité inférieure du cycle. L'angle $\psi$ a alors la signification indiquée sur la figure 14 . On obtient ainsi les réseaux de courbes des figures 15 et 16 . On remarque que les variations de $\sigma(\psi)$ à l'extrémité B du cycle sont approximativement symétriques des variations à l'extrémité $\mathrm{A}$.

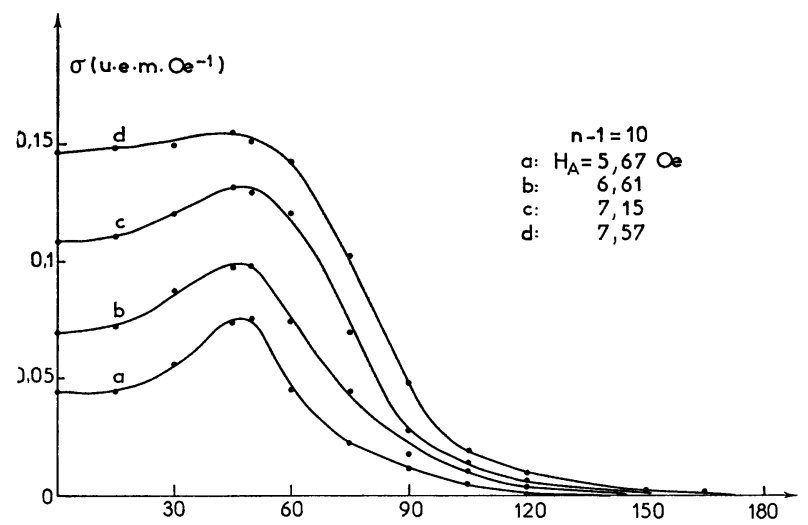

Fig. 15. - Variation angulaire de $\sigma(\psi)$ mesurée à l'extrémité B du cycle pour différentes valeurs du champ principal.

[Angular variation of $\sigma(\psi)$ measured at the lower corner B of the loop for different values of the main field.]

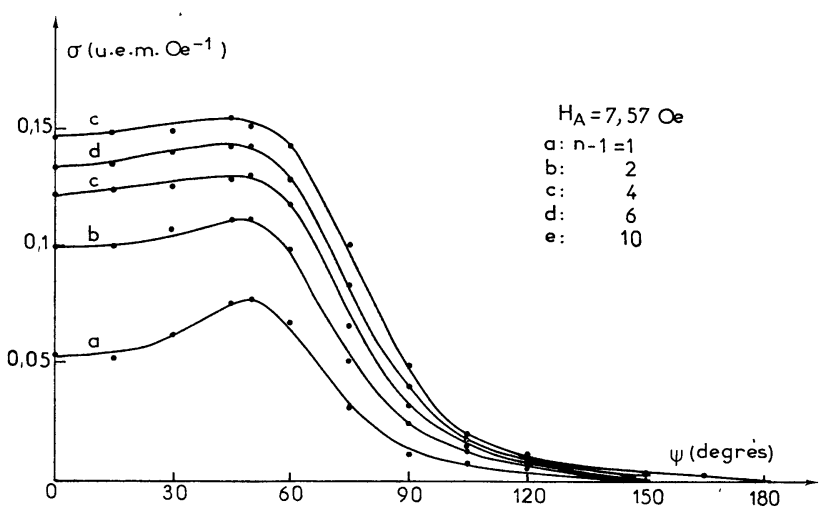

Fig. 16. - Variation angulaire de $\sigma(\psi)$ mesurée à l'extrémité B du cycle pour différentes valeurs du nombre de cycles.

[Angular variation of $\sigma(\psi)$ measured at the lower corner B of the loop for different numbers of cycles.]

\subsection{RéSULTATS RELATIFS A L'ÉCHANTILLON DE COBALT} EN PHASE CUBIQUE. - Nous avons mesuré pour cet échantillon un champ coercitif $H_{\mathrm{c}}=7,8$ Oe et une constante de traînage à la température ambiante $S=9,3$ mOe. Ici encore, nous avons constaté que la reptation devient prépondérante à partir de $H / H_{\mathrm{c}} \sim 0,3$. Le champ de reptation a été trouvé égal à $60,0 \mathrm{mOe}$ pour $H / H_{\mathrm{c}}=0,5$ et $n-1=10$.

La figure 17 représente les variations de $\Delta_{0}$ en fonction de l'angle $\Psi$ pour deux valeurs de $H_{\mathrm{A}}$ et $n-1=10$. On observe cette fois un maximum de $\Delta_{0}(\psi)$ voisin de $\psi=30^{\circ}$. Le même phénomène se reproduit pour les

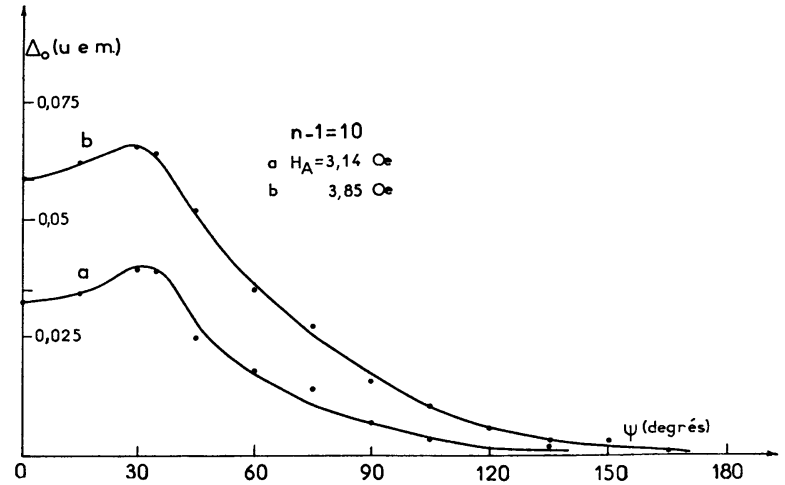

FIG. 17. - Variation angulaire de $\Delta_{0}(\psi)$ pour deux valeurs différentes du champ principal (échantillon de cobalt cubique). [Angular variation of $\Delta_{0}(\psi)$ for two different values of the main field (cubic cobalt).]

variations angulaires de $\sigma$ mesurées aux extrémités A et $\mathbf{B}$ des cycles.

\subsection{RÉSULTATS RELATIFS A L'ÉCHANTILLON DE COBALT} EN PHASE hEXAGONALE. - Son champ coercitif et sa constante de traînage valent respectivement $10,1 \mathrm{Oe}$ et $10,8 \mathrm{mOe}$. Nous avons constaté que les valeurs expérimentales de la quantité $\Delta_{0} / S_{\mathrm{AB}}$ restent toujours très voisines du terme de reptation thermique $S \log n$, et cela même dans les champs les plus intenses que peuvent fournir les bobines. Par exemple, pour $H_{\mathrm{A}} / H_{\mathrm{c}}=0,76$ et $n-1=10$, nous avons mesuré $\Delta_{0} / S_{\mathrm{AB}}=26,8 \mathrm{mOe}$ alors que l'on calcule

$$
S \log n=25,9 \mathrm{mOe} .
$$

En vue d'appliquer des champs plus élevés, nous avons préparé un tore à partir du disque en découpant sa partie centrale par électro-érosion, ce qui présente l'avantage de perturber au minimum l'échantillon.

Pour $H_{\mathrm{A}}=H_{\mathrm{c}}$, nous avons alors mesuré $\Delta_{0} / S_{\mathrm{AB}}=36,0 \mathrm{mOe}$. Cette valeur est encore assez proche de $S \log n$ pour qu'on puisse l'attribuer à un traînage prépondérant. L'expression (13) permet toutefois d'estimer l'ordre de grandeur du champ de reptation et l'on trouve $h_{\mathrm{r}} \sim 14,8 \mathrm{mOe}$, soit environ $1,5 \times 10^{-3} H_{\mathrm{c}}$. Dans les mêmes conditions $\left(H_{\mathrm{A}}=H_{\mathrm{c}}\right)$, on trouve un champ de reptation égal à $1,5 \times 10^{-2} H_{\mathrm{c}}$ pour un échantillon d'acier [8].

Il semble donc que seule la reptation thermique intervienne lorsque $H_{\mathrm{A}}$ ne dépasse pas 0,8 ou $0,9 H_{\mathrm{c}}$, Nous avons pu vérifier cette hypothèse grâce aux variations thermiques différentes des champs de traînage et de reptation qui sont respectivement des fonctions croissante et décroissante de la température [19,8]. A la température de l'azote liquide $(77 \mathrm{~K})$ et pour $H_{\mathrm{A}} / H_{\mathrm{c}}=0,76$ et $n-1=10$, nous avons mesuré $\Delta_{0} / S_{\mathrm{AB}}=10,5 \mathrm{mOe}$ alors que cette quantité valait 26,8 mOe à la température ambiante. Cela confirme le rôle essentiel du traînage de fluctuations thermiques et l'absence quasi totale du phénomène de reptation. D'ailleurs, J. C. Barbier [19] a montré qu'une loi en $T^{3 / 4}$ représente bien la variation thermique de la 
constante de traînage $S$. On peut donc s'attendre à un rapport :

$$
\frac{S_{77}}{S_{290}}=\left(\frac{77}{290}\right)^{3 / 4}=0,369,
$$

alors qu'on trouve expérimentalement

$$
\frac{10,5}{26,8}=0,392 \text {. }
$$

7. Interprétation des résultats expérimentaux. Dans le cadre de la théorie de L. Néel, nous avons cherché l'explication du phénomène de reptation dans des remaniements aléatoires de la structure en domaines. L'anisotropie de la reptation et sa disparition dans une substance magnétiquement uniaxe suggèrent une analogie avec une autre manifestation de l'hystérésis ferromagnétique qui est l'anisotropie de désaimantation $[12,14,18]$. Rappelons brièvement ce qu'on entend par ce terme : lorsqu'on a désaimanté un échantillon polycristallin par un champ magnétique alternatif de direction fixe et d'amplitude lentement décroissante, les propriétés du matériau dépendent de la direction suivant laquelle on les étudie. Ce phénomène est dû à une possibilité de choix au niveau de chaque cristallite entre plusieurs axes de facile aimantation qui ne sont plus énergiquement équivalents. En effet, la direction de facile aimantation la plus proche du champ de désaimantation est privilégiée. Les vecteurs aimantation spontanée ont tendance à se placer suivant cette direction. Il en résulte une répartition anisotrope de ces vecteurs qui entraîne une répartition anisotrope des domaines. Un tel phénomène disparaît dans une substance magnétiquement uniaxe puisqu'il n'y a alors plus de choix possible entre plusieurs directions.

Nous supposerons de même que la reptation est due à des remaniements aléatoires de la structure en domaines, rendus possibles par le fait qu'à une aimantation macroscopique donnée correspondent de très nombreuses configurations en domaines énergétiquement très voisines parmi lesquelles le système fait un choix aléatoire à chaque cycle. Cette situation suppose l'existence de plusieurs axes de facile aimantation et ne se rencontre jamais dans une substance uniaxe dont la structure en domaines reste figée.

Une telle hypothèse est étayée par les observations expérimentales de Daniel-Szabo et Gengnagel [20]. Ces auteurs étudient un monocristal de fer-aluminium taillé en forme de cadre dont les arêtes sont parallèles aux axes de facile aimantation du type $<100>$. Dans ces conditions, l'essentiel de la structure magnétique ne comprend que quelques parois de Bloch à $180^{\circ}$ mobiles, parallèles aux côtés du cadre et se raccordant dans les angles sur quatre parois à $90^{\circ}$ fixes. Aucun phénomène de reptation ne se manifeste. Au contraire, après avoir introduit des déformations locales dans l'échantillon, on observe une reptation importante. Nous proposons l'explication suivante : dans le monocristal non perturbé, l'aimantation spontanée garde toujours la même direction en un point donné et tout se passe comme si le matériau était magnétiquement uniaxe. Seuls interviennent des déplacements de parois sans modification de la topologie des domaines. Au contraire, les perturbations mécaniques font apparaître de nombreux domaines de fermeture et des parois à $90^{\circ}$, permettant ainsi l'occupation effective des trois directions de facile aimantation $\left({ }^{1}\right)$. Le choix de la direction la plus favorable en un point donné peut alors avoir lieu à chaque cycle et entraîne des remaniements dans la structure en domaines. De même, on peut admettre que la faible reptation observée sur l'échantillon de cobalt hexagonal est due à la petite proportion de matériau en phase cubique qui y subsiste. Nous allons donc essayer d'interpréter les résultats expérimentaux en faisant les hypothèses suivantes:

- Dans un matériau ayant plusieurs axes de facile aimantation, la structure en domaines peut se modifier d'un cycle à l'autre grâce à un choix possible entre plusieurs structures, d'énergies très voisines. Il faut vraisemblablement chercher l'origine de ces changements de configuration dans l'agitation thermique qui déclenche des sauts aléatoires entre divers états métastables [1]. Il en résulte, à chaque cycle, de nouvelles valeurs des interactions dans le matériau et l'aimantation évolue irréversiblement. La quantité $\Delta_{0}$ représente l'aimantation ainsi acquise.

- Du point de vue des mécanismes d'aimantation, on peut caractériser une paroi séparant deux domaines élémentaires orientés suivant $\mathbf{J}_{1}$ et $\mathbf{J}_{2}$ par le vecteur polarisation $\mathbf{p}=\mathbf{J}_{2}-\mathbf{J}_{1}$. A une structure en domaines déterminée correspondra une certaine répartition de vecteurs polarisation. Nous posons qu'au fil des cycles, la répartition des vecteurs polarisation évolue. Cette évolution est due à l'apparition de nouvelles parois dont l'orientation du vecteur polarisation par rapport au champ appliqué est plus favorable $\left({ }^{2}\right)$. Par consé-

(1) L'épaisseur du cadre étudié est très grande par rapport à l'épaisseur de paroi : quelques dixièmes de $\mathrm{mm}$ devant 1500 à $2000 \AA$. Les perturbations mécaniques consistent en des déformations plastiques locales obtenues à l'aide d'une pointe de diamant utilisée pour les mesures de micro-dureté. Il apparaît dans ces zones perturbées des domaines de fermeture dans lesquels l'aimantation peut être perpendiculaire au plan du cadre.

(2) Dans le domaine de Rayleigh proprement dit $\left(\sim H_{\mathrm{c}} / 10\right)$, le champ magnétique appliqué ne modifie pas de façon mesurable la répartition des vecteurs polarisation. Cette affirmation découle en particulier de l'étude de l'anisotropie de la désaimantation [18]. Elle a pour conséquence qu'il ne doit pas y avoir de reptation dans ce domaine de champs, ce qui est confirmé par l'expérience (Fig. 9). Cela ne signifie pas obligatoirement que le système de parois reste absolument immuable.

Au-delà du domaine de Rayleigh, et jusqu'à des champs de l'ordre de $H_{\mathrm{c}} / 2$, l'aimantation suit encore d'assez près une loi de Rayleigh. Pour les substances étudiées ici, l'écart ne dépasse pas quelques $10^{-2}$. Mais pour de telles variations de champ, il y a au cours des cycles disparition et création de parois. Ces deux faits ne sont pas incompatibles. En effet, d'une part, une paroi isolée obéit à une loi de Rayleigh [2] ; d'autre part, dans les champs considérés et pour les substances du type fer dont l'anisotropie est relativement élevée, la contribution des mécanismes de rotation est négligeable. 
quent, les caractéristiques directionnelles du matériau (susceptibilités) se modifient au cours de son histoire magnétique. La quantité $\sigma$ représente les variations de la susceptibilité irréversible par rapport à une valeur de référence mesurée à la première application du champ $H_{\mathrm{A}}$.

7.1 INTERPRÉTATION DES VARIATIONS DE $\sigma$. - Les $n$ applications successives du champ $H_{\mathrm{A}}$ ont modifié la répartition de polarisations qui caractérise la structure en domaines. Soit $S_{\mathrm{i}}^{n-1}$ la susceptibilité différentielle irréversible au point $\mathrm{A}$ correspondant à cette répartition. Elle diffère de $S_{\mathrm{i}}^{0}$ qui est celle que l'on mesure à la première application du champ car la structure en domaines a été modifiée entre temps. On a :

$$
\begin{aligned}
\delta J_{0} & =S_{\mathrm{i}}^{0} \delta H \\
\delta J_{n-1} & =S_{\mathrm{i}}^{n-1} H-\Delta_{0}
\end{aligned}
$$

Le terme $\Delta_{0}$ étant la variation d'aimantation irréversible due à la reptation au cours des $n$ applications du champ. La quantité $\Delta$ qui est la différence $\delta J_{0}-\delta J_{n-1}$ a pour expression :

$$
\Delta=\left(S_{\mathrm{i}}^{0}-S_{\mathrm{i}}^{n-1}\right) \delta H+\Delta_{0} .
$$

En identifiant avec l'expression (12), on tire :

$$
\sigma=S_{\mathrm{i}}^{0}-S_{\mathrm{i}}^{n-1} \text {. }
$$

Nous n'avons pas fait apparaître dans les expressions précédentes les termes non linéaires en $\delta H$ dus au fait que $\delta H$ n'est pas très petit devant $H_{\mathrm{A}}$.

Le problème consiste à remonter des variations de $\sigma$ à une répartition angulaire de vecteurs polarisation $\mathbf{p}$, caractérisant une répartition en domaines donnée. Un tel calcul est possible lorsque les déplacements de parois constituent le mécanisme d'aimantation prépondérant, c'est-à-dire dans la région des champs faibles. Pour le mener à bien, il est commode de remplacer une structure en domaines élémentaires par un ensemble de vecteurs polarisation dont chacun est affecté à une paroi [18]. Celui-ci détermine entièrement les variations d'aimantation dues au déplacement de la paroi dans un champ magnétique. Nous définissons une densité angulaire de vecteurs polarisation $f(\theta, \varphi)$. C'est cette densité qui est modifiée par les remaniements de la structure en domaines. Pour calculer les variations d'aimantation, nous supposerons que chaque direction de polarisation obéit à une loi de Rayleigh, c'est-à-dire que le déplacement de la paroi correspondante est régi par cette loi [18].

La figure 18 indique les notations employées dans le calcul. Le plan $x \mathrm{O} z$ est le plan du disque. L'axe $\mathrm{O} x$ est choisi suivant le champ principal $\mathbf{H}_{\mathrm{A}}$. Le champ additionnel $\boldsymbol{\delta} \mathbf{H}$ fait avec $\mathbf{H}_{\mathbf{A}}$ l'angle $\psi$ et on mesure les variations de flux dans la direction de $\delta \mathbf{H}$. Un vecteur polarisation $\mathbf{p}$ se projette en $\mathbf{p}^{\prime}$, sur le plan $z \mathrm{O} x$. On le repère par les angles $\varphi=(\mathrm{O} y, \mathbf{p})$ et $\theta=\left(\mathrm{O} x, \mathbf{p}^{\prime}\right)$. Le champ additionnel provoque un accroissement irré-

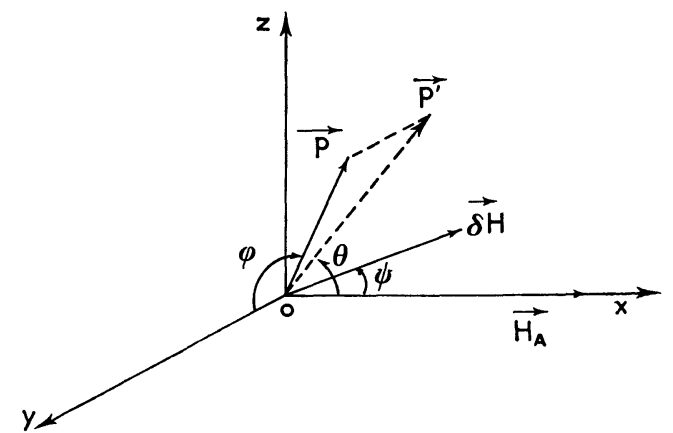

Fig. 18. - Notations employées dans le calcul de l'accroissement irréversible d'aimantation dû à l'application du champ additionnel.

[Symbols used in the calculation of the irreversible increase of magnetization due to the additional field.]

versible d'aimantation lorsque sa projection et celle de $\mathbf{H}_{\mathrm{A}}$, sur la direction de polarisation considérée, sont de même signe. Les vecteurs p qui satisfont à cette condition ont leur projection $\mathbf{p}^{\prime}$ comprise dans la région non hachurée de la figure 19. On voit que l'étude des susceptibilités irréversibles à l'extrémité $\mathrm{A}$ du cycle n'apporte des renseignements que sur la répartition des polarisations dans le demi-espace contenant $\mathbf{H}_{\mathrm{A}}$. Pour obtenir des informations sur l'autre demi-espace, il faut faire des mesures à l'extrémité $B$ du cycle.

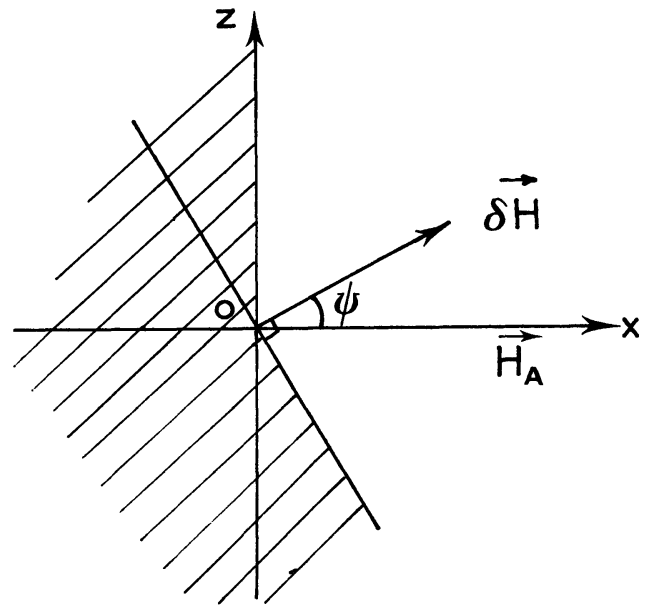

FIG. 19. - Directions de polarisation contribuant à l'accroissement irréversible d'aimantation.

[Polarization vectors which contribute to the irreversible increase of magnetization.]

En appliquant les lois de Rayleigh à la direction de polarisation $\mathrm{p}$, on trouve une contribution à l'aimantation irréversible :

$$
(\delta J)_{p}=2 b H_{\mathrm{A} p} \delta H_{p}+b \delta H_{p}^{2} .
$$

Dans cette expression, les symboles $H_{\mathrm{A} p}$ et $\delta H_{p}$, que nous appellerons champs effectifs, représentent les projections des champs $\mathbf{H}_{\mathrm{A}}$ et $\delta \mathbf{H}$ sur la direction consi- 
dérée. Nous ne calculerons que le terme linéaire en $\delta H_{p}$. Il est commode d'utiliser le tableau des cosinus directeurs des vecteurs intervenant dans le calcul :

\section{TABLEAU IV}

Cosinus directeurs des vecteurs $\mathbf{H}_{\mathrm{A}}, \boldsymbol{\delta} \mathbf{H}$ et $\mathbf{p}$ [Angular cosines of the vectors $\mathbf{H}_{\mathrm{A}}, \mathbf{\delta} \mathbf{H}$ and $\mathbf{p}$ ]

\begin{tabular}{lccl} 
& $\mathbf{H}_{\mathbf{A}}$ & $\boldsymbol{\delta H}$ & \multicolumn{1}{c}{$\frac{\mathbf{p}}{-}$} \\
$\mathrm{O} x$ & 1 & $\cos \Psi$ & $\cos \theta \sin \varphi$ \\
$\mathrm{O} y$ & 0 & 0 & $\cos \varphi$ \\
$\mathrm{O} z$ & 0 & $\sin \Psi$ & $\sin \theta \sin \varphi$
\end{tabular}

On trouve ainsi :

$$
(\delta J)_{p}=2 b H_{\mathrm{A}} \delta H \cos \theta \cos (\Psi-\theta) \sin ^{2} \varphi .
$$

Il faut ensuite projeter cette variation d'aimantation sur la direction de mesure, ce qui revient à la multiplier par la quantité $\cos (\Psi-\theta) \sin \varphi$. On introduit d'autre part la probabilité directionnelle normalisée $f(\theta, \varphi)$, représentant la densité de polarisations et on multiplie par l'élément d'intégration $\sin \varphi \mathrm{d} \varphi \mathrm{d} \theta$. La variation totale d'aimantation irréversible due à l'application de $\delta H$ se met finalement sous la forme :

$$
\begin{array}{r}
\delta J(\Psi)=2 b H_{\mathrm{A}} \delta H \int_{-\frac{\pi}{2}+\Psi}^{\frac{\pi}{2}} \cos \theta \cos ^{2}(\Psi-\theta) \mathrm{d} \theta \times \\
\times \int_{0}^{\frac{\pi}{2}} f(\theta, \varphi) \sin ^{4} \varphi \mathrm{d} \varphi .
\end{array}
$$

Le problème qui consiste à déterminer la fonction de deux variables $f(\theta, \varphi)$ n'a pas en général une solution unique puisqu'on connaît les variations de

$$
S_{\mathrm{i}}=\delta J(\Psi) / \delta H
$$

en fonction du seul paramètre angulaire $\Psi$. Il faudrait mesurer la susceptibilité irréversible dans toutes les directions de l'espace et pas seulement d'un plan. On remarque toutefois que le problème $a$ un axe de symétrie qui est la direction du champ principal $H_{\mathrm{A}}$. La fonction $f(\theta, \varphi)$ ne doit dépendre que de l'angle $\beta$ entre $H_{\mathrm{A}}$ et la direction de polarisation considérée, et doit être une fonction paire de $\beta$. C'est pourquoi nous exprimerons $f(\theta, \varphi)$ par un développement du type :

$$
f(\theta, \varphi)=\sum_{q=0}^{\infty} f_{q} \cos ^{q} \beta
$$

avec :

$$
\cos \beta=\cos \theta \sin \varphi .
$$

L'accroissement d'aimantation se met alors sous la forme :

$$
\delta J(\Psi)=\sum_{q=0}^{\infty} f_{q} I_{q}(\Psi)
$$

avec :

$$
\begin{array}{r}
I_{q}(\Psi)=2 b H_{\mathrm{A}} \delta H \int_{-\frac{\pi}{2}+\Psi}^{\frac{\pi}{2}} \cos ^{q+1} \theta \cos (\Psi-\theta) \mathrm{d} \theta \times \\
\times \int_{0}^{\frac{\pi}{2}} \sin ^{q+4} \varphi \mathrm{d} \varphi .
\end{array}
$$

Bien que cette expression ait l'allure d'un produit de convolution entre les variables $\theta$ et $\Psi$, la situation est un peu plus compliquée car les bornes d'intégration dépendent de $\Psi$. Il n'y a donc pas simplement multiplication des spectres de Fourier des deux fonctions convoluées. Ce problème a été traité sous son aspect le plus général par J. Bonnefous [21]. Pour le cas particulier qui nous intéresse ici, nous avons adopté une méthode de résolution approchée dont les détails sont donnés en annexe

7.1.1 Résultats pour $n-1=0$ - Ayant mesuré la fonction $\delta J(\Psi)$ à la première application du champ principal, on la décompose sous la forme :

$$
\delta J(\Psi)=2 b H_{\mathrm{A}} \delta H \sum_{r} \delta_{r} \cos ^{r} \Psi .
$$

On compare alors les taux d'harmoniques ainsi obtenus aux valeurs qu'ils auraient si la répartition des polarisations étaient isotrope. Rappelons que dans ce dernier cas, la variation angulaire $S_{\mathrm{i}}(\Psi)$ est proportionnelle à $(1+\cos \Psi)^{2}$ [12]. Le tableau 5 donne les résultats d'une telle décomposition dans le cas de l'échantillon d'acier pour un champ principal $H_{\mathrm{A}}=7,57$ Oe.

\section{TABleaU V}

Comparaison des valeurs expérimentales des rapports $\delta_{\mathrm{r}} / \delta_{0}$ avec leurs valeurs théoriques, calculées dans l'hypothèse d'une structure en domaines isotrope.

[Comparison between the experimental vcilues of the ratios $\delta_{\mathrm{r}} / \delta_{0}$ and their theoretical values calculated by assuming an isotropic domain structure].

$\begin{array}{llllccl}r & 0 & 1 & 2 & 3 & 4 & 5 \\ \delta_{\mathrm{r}} / \delta_{0} & 1 & 2,16 & 1,18 & -0,36 & -0,42 & 0,08 \\ \delta_{\mathrm{r}} / \delta_{0} \text { iso } & 1 & 2 & 1 & 0 & 0 & 0\end{array}$

La comparaison des deux lignes du tableau tend à prouver que la répartition des polarisations n'est déjà plus isotrope à la première application du champ. Ces écarts pourraient toutefois être dus au fait que le champ principal $H_{\mathrm{A}}$ est trop élevé et que les lois de Rayleigh ne sont plus valables. On sait que la susceptibilité irréversible croît alors plus vite que ne l'indique l'expression (18). Dans ces conditions, les directions de polarisation voisines de $H_{\mathrm{A}}$, soumises aux champs effectifs les plus importants, donnent des accroissements plus grands que ne le prévoit l'expression (19). Ces directions sont alors plus fortement pondérées dans l'intégration sur la fonction $f(\theta, \varphi)$ et la fonction $S_{\mathrm{i}}(\psi)$ 
est renforcée au voisinage de $\psi=0$. Cet effet devrait donc se traduire par une augmentation des coefficients $\delta_{r}$ à partir de $r=1$. On observe par contre que des termes fortement négatifs apparaissent aux ordres 3 et 4 , ce qui correspond à un renforcement de la susceptibilité dans des directions intermédiaires $(\psi \sim \pi / 3$ ou $\pi / 4)$. C'est pourquoi nous pensons que l'écart aux lois de Rayleigh, bien qu'on ne puisse l'exclure, n'est pas le phénomène prépondérant.

Nous admettrons donc que la première application du champ principal déforme la répartition en domaines initialement isotrope. Connaissant les valeurs expérimentales des coefficients $\delta_{r}$, on en déduit la densité angulaire des vecteurs polarisation $f_{0}(\theta, \varphi)$ suivant la méthode décrite en annexe (l'indice 0 rappelle que cette fonction est mesurée à la première application de $H_{\mathrm{A}}$, c'est-à-dire pour $n-1=0$ ). La figure 20 donne

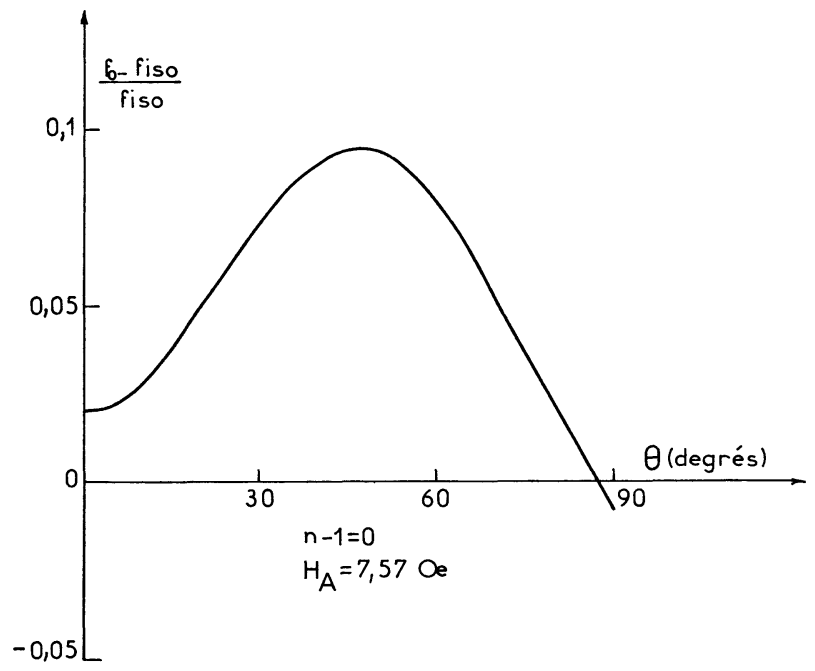

Fig. 20. - Déformation de la densité de polarisations $f(\theta, \varphi)$ à partir de l'état initial isotrope à la première application du champ principal (échantillon d'acier).

[Evolution of the polarization density $f(\theta, \varphi)$ from the initial isotropic state at the first application of the main field (steel sample).]

l'allure de $f_{0}(\theta, \varphi)$ dans la moitié du plan $x \mathrm{O} z$ (plan du disque) contenant le vecteur $H_{\mathrm{A}}$. Pour que la représentation soit plus nette, on a tracé en fait l'écart relatif avec la répartition isotrope. On voit que les modifications de la densité de polarisations atteignent $9 \%$ au voisinage de $\theta=45^{\circ}$. Cet enrichissement correspond aux harmoniques négatifs d'ordres 3 et 4 (Tableau V). Rappelons à ce propos qu'en étudiant l'anisotropie de désaimantation $[12,14,18]$, nous avons pu répartir l'ensemble des vecteurs polarisation en trois populations dénommées I, II, III suivant leurs orientations Nous avons constaté que les désaimantations unidirectionnelles d'amplitude inférieure à $H_{\mathrm{c}}$ favorisent la population II dont la densité angulaire de probabilité culmine autour de $45^{\circ}$. On observe ici un comportement analogue.
On peut remarquer de même que la figure 17 suggère que les directions favorisées dans l'échantillon de cobalt font avec $\mathbf{H}_{\mathbf{A}}$ un angle $\psi$ voisin de $30^{\circ}$. Les axes de facile aimantation du cobalt cubique $\left(K_{1}<0\right)$ sont les directions cristallographiques $\langle 111\rangle$. Le plus petit angle $\alpha$ entre ces directions vaut environ $70^{\circ}$.

On pourrait faire dans un polycristal de cobalt cubique supposé parfait une étude de la hiérarchie angulaire de ces directions par rapport à celle de $\mathbf{H}_{\mathrm{A}}$, tout à fait analogue à celle déjà faite [18] sur un polycristal où les axes de facile aimantation sont du type $<100>$ (type fer, $K_{1}>0$ ). On trouverait alors, entre autres, une population d'orientation moyennement favorable dont la densité de probabilité culminerait autour de $35^{\circ}$. Il semble que la figure 17 révèle un enrichissement de ces directions.

7.1.2 Résultats pour $n-1 \geqslant 1$. La fonction $\sigma(\psi)$ est la différence entre la susceptibilité irréversible $S_{\mathrm{i}}^{0}(\psi)$, mesurée à la première application du champ et la susceptibilité $S_{\mathrm{i}}^{n-1}(\psi)$ mesurée au $n^{\mathrm{e}}$ cycle. Grâce à la méthode de résolution décrite en annexe, on a accès aux modifications que subit, à chaque cycle, la fonction $f(\theta, \varphi)$ à partir de la répartition initiale $f_{0}(\theta, \varphi)$, obtenue à la première application du champ. Rappelons que cet état initial est lui-même anisotrope. La figure 21 représente la variation de $f_{n-1}(\theta, \varphi)$ pour $n-1=10$

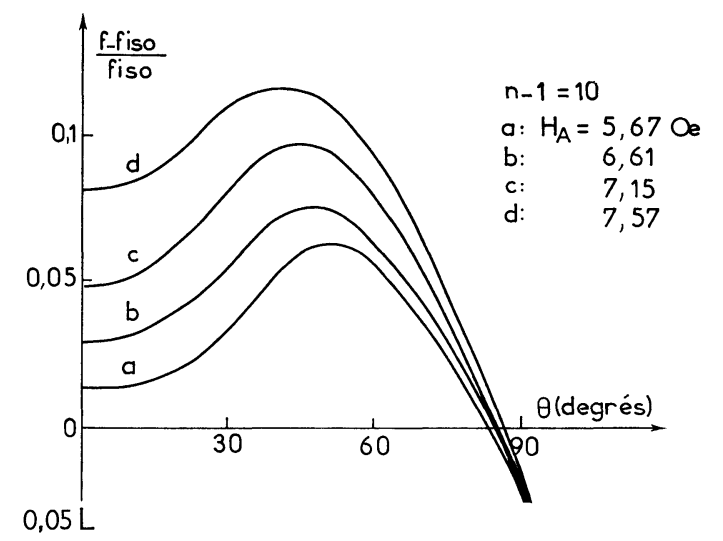

Fig. 21. - Evolution de la densité de polarisations $f(\theta, \varphi)$ dans le demi-espace contenant $\boldsymbol{H}_{\mathrm{A}}$ en fonction du champ principal (échantillon d'acier).

[Evolution of the polarization density $f(\theta, \varphi)$ in the half-space which contains $H_{\mathrm{A}}$, as a function of the magnitude of the main field (steel sample).]

et pour quatre valeurs différentes de $H_{\mathrm{A}}$. On constate que l'enrichissement des directions intermédiaires devient relativement moins important lorsque $H_{\mathrm{A}}$ augmente. La surface indicatrice de $f_{n-1}(\theta, \varphi)$ a tendance à s'allonger de plus en plus le long du vecteur $H_{\mathrm{A}}$.

La figure 22 montre l'évolution de la densité de vecteurs polarisation en fonction du nombre de cycles pour un champ donné $\left(H_{\mathrm{A}}=7,57 \mathrm{Oe}\right)$. On remarque que la structure en domaines évolue rapidement au cours des tout premiers cycles et beaucoup plus lentement ensuite. 


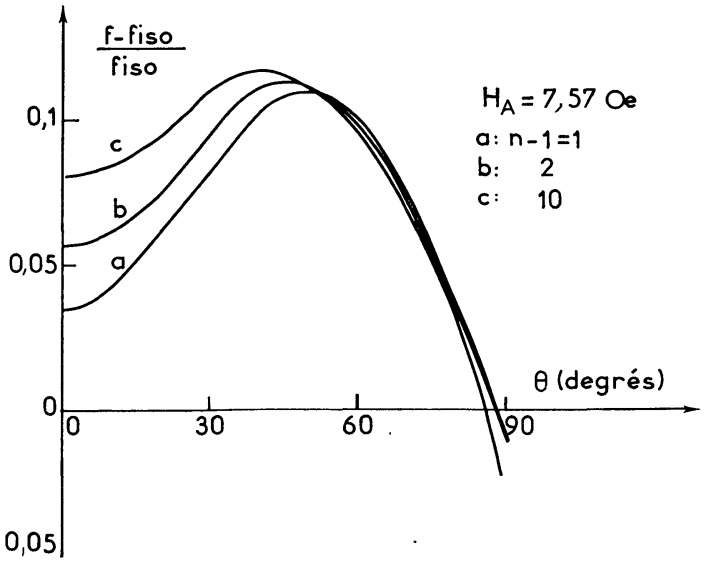

Fig. 22. - Evolution de la densité de polarisations $f(\theta, \varphi)$ dans le demi-espace contenant $H_{\mathrm{A}}$ en fonction du nombre de cycles (échantillon d'acier).

[Evolution of the polarization density $f(\theta, \varphi)$ in the half-space which contains $H_{\mathrm{A}}$, as a function of the number of cycles (steel sample).]

Tous les résultats ci-dessus étaient relatifs à l'extrémité $\mathrm{A}$ du cycle et montraient une augmentation progressive de la densité de polarisations dans le demiespace contenant $H_{\mathrm{A}}$. Les mesures de $\sigma$ à l'extrémité B révèlent au contraire un appauvrissement de l'autre demi-espace. Pour des valeurs données du champ et du nombre de cycles, on trouve que les modifications de $f(\theta, \varphi)$ dans chacun des demi-espaces sont à peu près symétriques l'une de l'autre. Les différences qui subsistent ne proviennent pas seulement des erreurs expérimentales. Elles sont également dues au fait que les mesures de $\sigma(\psi)$ aux extrémités A et B du cycle sont séparées par une décroissance du champ qui modifie légèrement la densité de vecteurs polarisation. Cette opération détruit en partie la répartition que l'on avait obtenue à l'extrémité $\mathrm{A}$. C'est la raison pour laquelle on ne peut obtenir une connaissance complète de la fonction $f(\theta, \varphi)$ à un instant donné par des mesures de susceptibilité irréversible. La figure 23 donne néanmoins une représentation imagée de $f_{n-1}(\theta, \varphi)$ en fonction du nombre de cycles. On a employé ici les coordonnées polaires et les écarts à l'isotropie ont été multipliés par 3 pour la clarté de la figure.

La même difficulté de principe apparaît lorsqu'on s'intéresse aux susceptibilités composées $S_{\mathrm{AB}}$ et $S_{\mathrm{BA}}$ dont la mesure implique deux séquences différentes d'application des champs (5 et 6). Rappelons que Nguyen Van Dang [8] a trouvé expérimentalement une différence de 15 à $30 \%$ entre ces deux quantités lorsque l'amplitude des cycles est égale à $H_{\mathrm{c}}$, alors que les divers modèles d'hystérésis prévoient $S_{\mathrm{AB}}=S_{\mathrm{BA}}$. Le désaccord provient de ce qu'on calcule habituellement les susceptibilités composées en supposant que les variations du champ appliqué ne modifient pas les propriétés statistiques du matériau.

De ce point de vue, le domaine de Rayleigh n'est pas seulement une région où l'aimantation suit une certaine

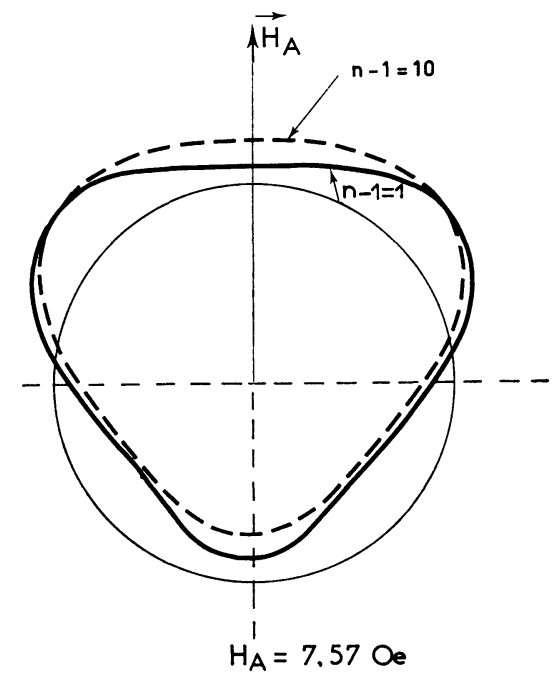

Fig. 23. - Evolution qualitative de la densité de polarisations $f(\theta, \varphi)$ dans tout l'espace en fonction du nombre de cycles (échantillon d'acier).

[Qualitative evolution of the polarization density $f(\theta, \varphi)$ in the whole space as a function of the number of cycles (steel sample).]

loi analytique. La limite du domaine de Rayleigh correspond à un seuil au-dessous duquel le champ magnétique ne parvient pas à perturber la répartition des vecteurs polarisation correspondant à la structure en domaines initiale.

7.2 INTERPRÉtation Des VARIATIONS De $\Delta_{0} .-\mathrm{Ce}$ paramètre représente l'aimantation irréversible qui a été acquise sous l'effet du champ aléatoire de reptation et qui ne peut donc pius être acquise par application d'un champ additionnel. Le principe du calcul de $\Delta_{0}(\psi)$ est le même que celui de $\delta J(\psi)$. Il faut tout d'abord calculer l'accroissement élémentaire d'aimantation sur une direction de polarisation repérée par ses paramètres angulaires $\theta$ et $\varphi$. On intègre ensuite sur toutes les directions de polarisation actives.

Le problème physique qui se présente alors consiste à connaître la répartition directionnelle du champ aléatoire de reptation caractérisé par ses moyennes spatiales des premier et second ordres (cf. § 2.2). Dans une structure en domaines complexe, il est logique d'admettre que $\langle\delta H\rangle$ est nul suivant toutes les directions de l'espace. Par contre, dans une répartition en domaines anisotropes, $\left\langle\delta H^{2}\right\rangle=h_{r}^{2}$ peut, a priori dépendre de la direction considérée. On remarque toutefois que la décroissance en $r^{-2}$ du champ créé par une charge ponctuelle est assez lente pour qu'en un point quelconque du matériau se fasse sentir l'influence de nombreux domaines. Par conséquent, les effets directionnels doivent être assez fortement amortis. D'autre part, nous n'avons pas pu mettre en évidence avec certitude, l'anisotropie d'un autre champ aléatoire qui est le champ de fluctuations thermiques responsable du traînage irréversible [1]. Nous supposerons donc en première approximation que le champ de 
reptation est isotrope et nous confronterons les conséquences d'une telle hypothèse avec les résultats expérimentaux.

On exprime la variation angulaire de $\Delta_{0}$ par une intégrale analogue à (20). La différence est due au fait que le champ additionnel est maintenant colinéaire à la direction de polarisation, ce qui supprime une opération de projection. En prenant la susceptibilité composée $S_{\mathrm{AB}}$ égale à $b H_{\mathrm{A} p}$ sur chaque direction de polarisation (cf. $\S 2.1$ ), on trouve :

$$
\begin{array}{r}
\Delta_{0}(\psi)=b H_{\mathrm{A}} x_{n} h_{r} \int_{-\frac{\pi}{2}+\psi}^{\frac{\pi}{2}} \cos \theta \cos (\psi-\theta) \mathrm{d} \theta \times \\
\times \int_{0}^{\frac{\pi}{2}} f(\theta, \varphi) \sin ^{3} \varphi \mathrm{d} \varphi .
\end{array}
$$

En décomposant $f(\theta, \varphi)$ sous la forme (21), on ramène le problème au calcul d'intégrales du type :

$$
\begin{array}{r}
I_{q}^{\prime}(\psi)=f_{q} \int_{-\frac{\pi}{2}+\psi}^{\frac{\pi}{2}} \cos ^{q+1} \theta \cos (\psi-\theta) \mathrm{d} \theta \times \\
\times \int_{0}^{\frac{\pi}{2}} \sin ^{q+3} \varphi \mathrm{d} \varphi .
\end{array}
$$

A cette étape du calcul apparaît une difficulté de principe. On a vu (§7.1.2) que la densité angulaire de polarisations $f(\theta, \varphi)$ se modifie à chaque cycle. On n'a donc pas le droit de calculer $\Delta_{0}(\psi)$ au $n^{\mathrm{e}}$ cycle en donnant aux coefficients $f_{q}$ des valeurs fixes. Il faut exprimer $\Delta_{0}(\psi)$ comme la somme des accroissements irréversibles d'aimantation acquis à chaque cycle de rang $m \leqslant n$ grâce au champ de reptation agissant sur la répartition qui existait à cet instant. Soient $f_{q m}$ les coefficients du développement de $f(\theta, \varphi)$ au cycle de rang $m$. Le problème revient à calculer :

$$
\begin{gathered}
\Delta_{0}(\psi)=b H_{\mathrm{A}} h_{r} \sum_{m=1}^{n}\left(x_{m}-x_{m-1}\right) \\
\sum_{q} f_{q m} \int_{-\frac{\pi}{2}+\psi}^{\frac{\pi}{2}} \cos ^{q+1} \theta \cos (\psi-\theta) \mathrm{d} \theta \int_{0}^{\frac{\pi}{2}} \sin ^{q+3} \varphi \mathrm{d} \varphi
\end{gathered}
$$

expression qui se ramènerait à (26) si les $f_{q m}$ étaient indépendants de $m$.

Nous avons effectué ce calcul pour $n-1=4$ et $H_{\mathrm{A}}=7,57 \mathrm{Oe}$, en prenant pour $f(\theta, \varphi)$ l'évolution schématisée par la figure 22. La figure 24 donne les résultats : on constate que les variations angulaires des courbes expérimentale et calculée sont sensiblement les mêmes. Nous en conclurons que l'hypothèse d'isotropie du champ de reptation, si elle n'est pas rigoureuse, représente néanmoins une bonne approximation compte tenu de l'incertitude expérimentale.

Le calcul précédent tient compte de l'évolution de $f(\theta, \varphi)$ avec le nombre de cycles. Nous pensons que l'on peut expliquer ainsi le fait que le rapport $\Delta_{0} / S_{\mathrm{AB}} x_{n}$ ne soit pas constant (Tableau III). L'enrichissement de la densité de polarisations suivant des directions

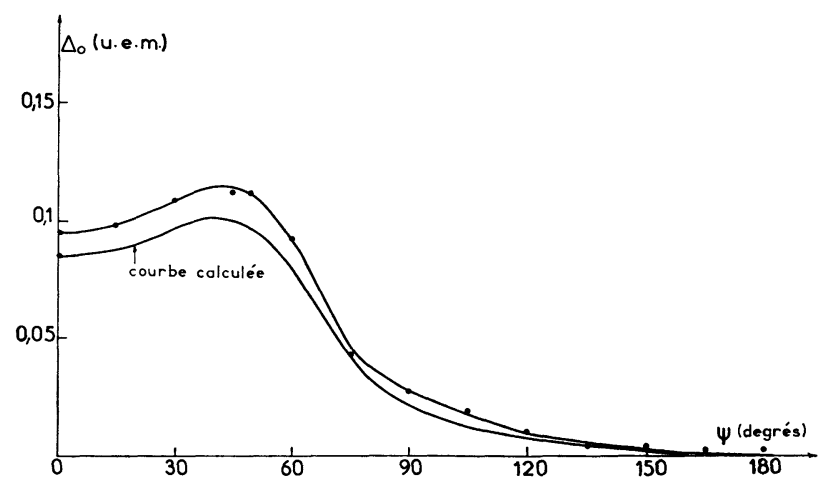

Fig. 24. - Variations angulaires expérimentale et calculée de la quantité $\Delta_{0}(\psi)\left(n-1=4 ; H_{\mathrm{A}}=7,57 \mathrm{Oe}\right.$; échantillon d'acier). [Measured and calculated angular variations of $\Delta_{0}(\psi)$ $\left(n-1=4 ; H_{\mathrm{A}}=7.57 \mathrm{Oe} ;\right.$ steel sample $\left.).\right]$

voisines de $H_{\mathrm{A}}$ rend de plus en plus efficace l'action du champ de reptation. Les valeurs de celui-ci paraissent croître si l'on ne tient pas compte de l'adaptation progressive du matériau à un certain processus d'aimantation.

On remarque également sur la figure 24 que la courbe calculée est entièrement au-dessous de la courbe expérimentale. Si on admet que cet écart n'est pas entièrement dû aux approximations introduites dans le calcul, on pourrait en proposer l'explication suivante. Le calcul suppose pour simplifier que l'évolution de toutes les parois a pour origine commune la première application du champ principal. En réalité, les réorganisations continuelles du matériau ont fait apparaître à chaque cycle de rang inférieur ou égal à $n$, de nouvelles parois qui n'avaient pas encore été soumises au champ aléatoire de reptation. Plus précisément, soit $m$ le rang du cycle où a été créée une paroi : l'évolution de celle-ci aux cycles ultérieurs de rang $m^{\prime}$ est alors gouvernée, non par la quantité $x_{m^{\prime}}$, mais par $x_{m^{\prime}-m}$. Or, les variations de cette quantité sont les plus rapides pour les faibles valeurs de l'indice (Tableau I). Par conséquent, les parois créées au cours des cycles successifs évoluent plus vite que celles qui existaient à la première application du champ principal, et cela d'autant plus que leur apparition est plus récente. Il en résulte une croissance de $\Delta_{0}(\psi)$ en fonction du nombre de cycles plus rapide que ne le prévoit l'expression (28).

8. Conclusion. - En étudiant la reptation des cycles de champ sur des échantillons polycristallins, nous avons constaté que le comportement directionnel de ce phénomène fournit des renseignements précieux pour son interprétation. Au cours des cycles, la répartition angulaire des vecteurs polarisation se modifie et s'adapte progressivement à un certain processus d'aimantation. Une telle réorganisation n'est possible que si la substance possède plusieurs axes de facile aimantation, et c'est pourquoi la reptation disparaît dans une substance magnétiquement uniaxe. On voit ainsi réapparaître à notre échelle la symétrie microscopique du matériau. 
La reptation ne doit pas être considérée comme un aspect marginal de l'hystérésis. Elle apparaît dès que sont réalisées les conditions suivantes : des couplages doivent se manifester dans la structure en domaines, le système magnétique doit pouvoir adopter de nombreuses configurations d'énergies voisines, et il doit pouvoir passer aléatoirement de l'une à l'autre. A ce sujet, nous avons pu montrer [2] qu'un système de deux parois est déjà le siège de fortes interactions magnétostatiques conduisant à deux états stables différents entre lesquels l'agitation thermique permet des sauts aléatoires. On peut raisonnablement penser qu'un matériau complexe présente a fortiori le même comportement. Toutefois, l'analogie n'est pas complète : on a vu [1] que des échantillons monocristallins à structures en domaines simples ne donnent pas lieu au phénomène de reptation, bien que le déplacement des parois fasse intervenir une part de hasard, parce que la topologie des domaines ne change pas. Un mécanisme de traînage de fluctuations suffit alors à décrire les accroissements aléatoires d'aimantation irréversible. De même, la figure 9 montre que la reptation disparaît lorsque l'amplitude des cycles devient trop faible pour pouvoir modifier la répartition en domaines élémentaires. Remarquons au passage que le domaine de Rayleigh est donc une région où l'on peut étudier le traînage de fluctuations thermiques et son anisotropie dans une structure en domaines donnée, sans que la superposition de deux phénomènes différents vienne compliquer l'interprétation des résultats [1].

$\mathrm{La}$ reptation révèle une évolution progressive du matériau vers un état de plus en plus ordonné. On peut se demander quelle est la limite de cette évolution. Il semble qu'une bonne approximation soit l'état d'aimantation anhystérétique obtenu en superposant au champ principal $H_{\mathrm{A}}$ un champ alternatif dont l'amplitude, initialement grande, décroît lentement jusqu'à zéro. Chaque région du matériau est ainsi soumise à toutes les valeurs possibles du champ magnétique et acquiert l'aimantation irréversible maxima compatible avec le champ $H_{\mathrm{A}}$. On peut citer à l'appui de cette hypothèse, une observation de Lliboutry [6] d'après laquelle la reptation disparaît lorsque l'échantillon est dans l'état anhystérétique. En adoptant pour la reptation le comportement asymptotique en $(\log n)^{1 / 2}$, on trouve alors [1] qu'il faudrait décrire au moins $10^{22}$ cycles pour atteindre la limite. Nous n'avons donc accès expérimentalement qu'aux tout premiers stades de la réorganisation du matériau.

\section{ANNEXE}

Calcul des modifications de la densité de vecteurs polarisation $f(\theta, \varphi)$ à partir des variations angulaires de la quantité $\sigma(\psi)$.

1. CAS DE LA PREMière APPLICATION DU ChAMP PRINCIPAL $(n-1=0)$. - Les intégrales $I_{q}$ (24) sont des fonctions des puissances successives de $\cos \Psi$ et $\sin \Psi$. Par suite de la symétrie du problème par rapport à $\Psi=0$, les puissances impaires de $\sin \Psi$ s'annulent et on peut ramener $I_{q}$, à une somme des puissances de $\cos \Psi$ que nous écrirons :

$$
I_{q}=2 b H_{\mathrm{A}} \delta H \sum_{r} a_{q r} \cos ^{r} \Psi .
$$

Par exemple, le calcul de la susceptibilité irréversible pour un état désaimanté isotrope ne fait intervenir que la quantité $f_{0} I_{0}$, les $f_{q}$ étant tous identiquement nuls pour $q \geqslant 1$. On trouve que $f_{0} I_{0}$ est égal à $\frac{(1+\cos \Psi)^{2}}{16}$ et on en déduit dans ce cas [12] :

$$
\frac{\delta J(\Psi)}{\delta H}=S_{\mathrm{i}}^{\mathrm{iso}}(\Psi)=\frac{b H_{\mathrm{A}}}{8}(1+\cos \Psi)^{2} .
$$

Alors que l'indice $q$ ne prend ici que la valeur 0 , l'indice $r$ prend les valeurs 0,1 et 2 .

A la suite de cette opération, la variation irréversible d'aimantation devient :

$$
\delta J(\Psi)=2 b H_{\mathrm{A}} \delta H \sum_{q=0}^{\infty} f_{q} \sum_{r} a_{q r} \cos ^{r} \Psi
$$

qui, en posant :

$$
\sum_{q=0}^{\infty} f_{q} a_{q r}=\delta_{r}
$$

se met sous la forme déjà rencontrée :

$$
\delta J(\Psi)=2 b H_{\mathrm{A}} \delta H \sum_{r} \delta_{r} \cos ^{r} \Psi .
$$

Ces manipulations n'ont pour but que de regrouper toutes les contributions à la susceptibilité en $\cos ^{r} \psi$ quel que soit le degré du monôme $f_{q} \cos ^{q} \beta$ qui leur a donné naissance. On voit que la difficulté essentielle du problème provient de ce que chaque terme du développement de $\delta J(\psi)$ résulte d'une sommation sur tous les termes du développement de $f(\theta, \varphi)$.

Nous avons recherché une solution approchée en faisant les remarques suivantes. D'une part, on peut raisonnablement admettre que les coefficients $f_{q}$ du développement de $f(\theta, \varphi)$ décroissent assez rapidement en fonction de $q$. Nous avons d'ailleurs montré en étudiant l'anisotropie de désaimantation [18] que les harmoniques présents dans la fonction $f(\theta, \varphi)$ sont notables jusqu'à l'ordre $q=2$ inclus. Ils sont par contre nettement plus faibles et même pratiquement nuls dans certaines conditions pour $q \geqslant 4$.

D'autre part, les quantités $I_{q}$ décroissent rapidement en fonction de $q$ car elles proviennent de l'intégration de fonctions trigonométriques à des puissances croissantes. Cela revient à remarquer qu'une mesure de susceptibilité fait intervenir toutes les directions de polarisation contenues dans un angle solide important. Par conséquent, les variations angulaires de la susceptibilité $S_{\mathrm{i}}(\Psi)$ ne reflètent que d'une façon très amortie les accidents de la densité $f(\theta, \varphi)$. Expérimentalement, on peut déterminer les harmoniques de $S_{\mathrm{i}}(\psi)$ avec une 
précision convenable jusqu'à l'ordre 4 inclus (Tableau V).

C'est pourquoi, nous avons limité en pratique les indices $q$ et $r$ à 4 et nous avons adopté la méthode de résolution suivante. Ayant obtenu les valeurs des coefficients $\delta_{r}$ jusqu'à l'indice 4 inclus, par décomposition de la fonction expérimentale $\delta J(\psi)(25)$, on écrit le système linéaire (32) sous la forme approchée

$$
\delta_{r}=\sum_{q=0}^{4} f_{q} a_{q r}
$$

où les quantités $a_{a r}$ sont données par le calcul. On en déduit les coefficients $f_{q}$ par inversion de la matrice $a_{q r}$, ce qui permet d'obtenir une approximation de la fonction $f(\theta, \varphi)$ sous la forme :

$$
f(\theta, \varphi)=\sum_{q=0}^{4} f_{q} \cos ^{q} \beta .
$$

On peut remarquer que l'opération physique de mesure d'une susceptibilité fait intervenir une sorte de filtrage sur les fréquences angulaires contenues dans la fonction $f(\theta, \varphi)$. La méthode décrite ci-dessus ne représente en définitive qu'un procédé approximatif de déconvolution de la fonction $S_{\mathrm{i}}(\psi)$ connaissant l'opérateur de filtrage. Comme toutes les méthodes de ce type, elle exige une précision suffisante sur la mesure des quantités $S_{\mathrm{i}}$. C'est pourquoi il est essentiel de disposer d'un fluxmètre extrêmement sensible et précis pour aborder de telles études.

Rappelons également que la mesure des susceptibilités irréversibles ne permet de connaître la répartition des polarisations que dans le demi-espace vers lequel s'est produite la dernière variation du champ principal. Il faut donc refaire un calcul analogue pour l'extré- mité B du cycle. Les développements obtenus pour la fonction $f(\theta, \varphi)$ ne sont donc en toute rigueur que des approximations valables sur un angle solide égal à $2 \pi$ et non pas de véritables décompositions sur un ensemble de fonctions. Il n'en serait pas de même si on mesurait la susceptibilité réversible à laquelle contribuent toutes les directions de polarisation mais l'étude de la susceptibilité irréversible reste préférable pour trois raisons :

- Elle a l'avantage de donner accès simultanément aux modifications de la structure en domaines (paramètre $\sigma$ ) et au terme de reptation qui en résulte (paramètre $\Delta_{0}$ ).

- Elle permet de tenir compte du traînage par un minutage approprié des expériences.

- Enfin, la figure 23 montre que les contributions des deux demi-espaces aux variations de la susceptibilité irréversible se compenseraient l'une l'autre en grande partie, augmentant sensiblement l'incertitude expérimentale.

2. Calcul de $f(\theta, \varphi)$ Au $n^{\text {e }}$ CYCLe. - La pente $\sigma(\psi)$ étant égale à la différence :

$$
S_{\mathbf{i}}^{0}(\psi)-S_{\mathbf{i}}^{n-1}(\psi),
$$

il suffit de remplacer le système linéaire (33) par :

$$
\delta_{r}-\sigma_{r}=\sum_{q=0}^{4} f_{q} a_{q r}
$$

où les quantités $\sigma_{r}$ sont les coefficients du développement de $\sigma(\psi)$ :

$$
\sigma(\psi)=2 b H_{\mathrm{A}} \sum_{r} \sigma_{r} \cos ^{r} \psi
$$

expression analogue à (25).

\section{Bibliographie}

[1] PorteseIL, J. L., Thèse, Grenoble (1975).

[2] Porteseil, J. L., Vergne, R., J. Physique 37 (1976) 929.

[3] Porteseil, J. L., Vergne, R., J. Physique 37 (1976) 1211.

[4] EwING, J. A., Phil. Trans. 176 (1885) 569.

[5] Bouasse, H., Berthier, Ann. Chim. Phys. 10 (1907) 199.

[6] Lliboutry, L., Ann. Phys. 6 (1951) 731.

[7] Barbier, J. C., Ann. Phys. 9 (1954) 84.

[8] NGuYen van Dang, Thèse, Grenoble (1961).

[9] Neet, L., C. R. Hebd. Séan. Acad. Sci. 244 (1945) 2668.

[10] Nées, L., Proc. R. A. Welch Foundation Conf. Chem. Res. II, Atomic Structure, Houston, Texas (1958).

[11] NéEL, L., J. Physique Rad. 12 (1951) 339.
[12] Vergne, R., BlazeK, Z., Porteseil, J. L., Phys. Stat. Sol. (a) 21 (1974) 141.

[13] Moissan, M. L., Diplôme d'Etudes Supérieures, Grenoble.

[14] Vergne, R., Porteseil, J. L., Blazek, Z., Phys. Stat. Sol. (a) 25 (1974) 171.

[15] BlazeK, Z., Thèse Docteur-Ingénieur, Grenoble, 1972.

[16] Vergne, R., PorteseiL, J. L., Revue Phys. Appl. 6 (1971) 95.

[17] Rimet, G., Thèse, Grenoble (1967).

[18] Vergne, R., Porteseil, J. L., Blazek, Z., Revue Phys. Appl. 10 (1975) 195.

[19] BARBIER, J. C., Thèse, Grenoble (1953).

[20] Daniel-Szabo, J., Gengnagel, H., Phys. Stat. Sol. 1 (1961) 512.

[21] Bonnefous, J., Acta Electronica 11 (1968) 7. 